\title{
Boundary Conditions for Coupled Quasilinear Wave Equations with Application to Isolated Systems
}

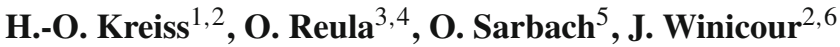 \\ 1 NADA, Royal Institute of Technology, 10044 Stockholm, Sweden \\ 2 Albert Einstein Institute, Max Planck Gesellschaft, Am Mühlenberg 1, \\ D-14476 Golm, Germany \\ 3 FaMAF Universidad Nacional de Córdoba, Córdoba, Argentina 5000 \\ 4 Instituto de Física Enrique Gaviola, CONICET, Córdoba, Argentina \\ 5 Instituto de Física y Matemáticas, Universidad Michoacana de San Nicolás de Hidalgo, \\ Edificio C-3, C.P. 58040 Morelia, Michoacán, Mexico. E-mail: sarbach@ifm.umich.mx \\ 6 Department of Physics and Astronomy, University of Pittsburgh, Pittsburgh, \\ Pennsylvania 15260, USA
}

Received: 21 July 2008 / Accepted: 8 December 2008

Published online: 18 April 2009 - (C) Springer-Verlag 2009

\begin{abstract}
We consider the initial-boundary value problem for systems of quasilinear wave equations on domains of the form $[0, T] \times \Sigma$, where $\Sigma$ is a compact manifold with smooth boundaries $\partial \Sigma$. By using an appropriate reduction to a first order symmetric hyperbolic system with maximal dissipative boundary conditions, well posedness of such problems is established for a large class of boundary conditions on $\partial \Sigma$. We show that our class of boundary conditions is sufficiently general to allow for a well posed formulation for different wave problems in the presence of constraints and artificial, nonreflecting boundaries, including Maxwell's equations in the Lorentz gauge and Einstein's gravitational equations in harmonic coordinates. Our results should also be useful for obtaining stable finite-difference discretizations for such problems.
\end{abstract}

\section{Introduction and Main Results}

Motivated in part by the numerical computation of spacetimes on a finite domain with artificial boundaries, the initial-boundary value problem (IBVP) in general relativity has started to receive a lot of attention during the last few years (see [1] for a review). A well posed IBVP for Einstein's vacuum field equations was formulated for the first time by Friedrich and Nagy [2] based on tetrad fields and the theory of quasilinear, symmetric hyperbolic systems with maximal dissipative boundary conditions [3-5]. More recently, Kreiss and Winicour [6] formulated a well posed IBVP for the harmonic gauge formulation of the Einstein vacuum equations which casts the field equations into a set of ten coupled quasilinear wave equations subject to four constraints. There are two key ideas behind the result of [6]. The first one is the realization that the wave equations, when viewed as first order pseudodifferential equations, have a non-characteristic boundary matrix. This allows application of the boundary value theory for such systems developed by Kreiss in the 1970's [7]. The second idea is the formulation of boundary conditions for the frozen coefficient form of the harmonic Einstein equations which ensures constraint propagation and satisfies the estimates required by the Kreiss theory. The well posedness 
of the system and the generalization to the quasilinear case can then be established using the theory of pseudodifferential operators (see, for instance, [8]).

In a subsequent paper [9], similar results were obtained via more mundane energy estimates which follow by integration by parts, without resort to the pseudodifferential calculus. For this, a non-standard energy norm is constructed which is based upon the choice of a particular time-like direction adapted to the boundary conditions being imposed. With respect to this energy the Kreiss-Winicour boundary conditions are maximal dissipative and so standard well posedness theorems apply even in the quasilinear case $[5,10]$. Besides being a simpler proof, or at least a proof that can be followed completely by a reader not familiar with the pseudodifferential techniques, it implies similar results for the stability of finite difference approximations to Einstein's equations in the harmonic gauge. This follows from considering the semidiscrete system of ordinary differential equations in time obtained by substituting finite differences for spatial derivatives. If the semidiscrete system is stable, then for appropriate time discretizations the fully discrete system is guaranteed to be stable [11]. The stability of the semidiscrete system can be established by the use of finite difference operators satisfying summation by parts [12], the counterpart of integration by parts, by mimicking the steps leading to the continuum energy estimate. A summation by parts algorithm based upon the standard energy norm for the harmonic Einstein problem was developed in [13] and verified to be stable in numerical tests [14]. The non-standard energy norm employed here and in [9] provides the basis to formulate a summation by parts algorithm whose numerical stability follows from established theory.

In this paper we present a more general and geometric version of the foregoing results which applies to coupled systems of quasilinear wave equations with a certain class of boundary conditions. The strong well posedness of the resulting IBVP is established by reducing the wave system to first order symmetric hyperbolic equations subject to maximal dissipative boundary conditions. This allows us to identify the structure in first order systems which can be used to establish boundary stability. This structure arises from the non-absolute nature of time in Lorentzian physics, whereby a Lorentz boost gives rise to a new conserved energy and so to a different symmetrizer. ${ }^{1}$ Realizing this, we are able to restate and prove our earlier results in terms of standard maximal dissipative boundary conditions for symmetric hyperbolic systems.

As we show, our class of boundary conditions is sufficiently flexible for obtaining well posed IBVP formulations for different models of isolated systems in physics, including the wave equation, Maxwell's equations and the Einstein field equations. In what follows, we present the main mathematical result in Sect. I A with two applications in Sects. I B and I C. The corresponding proof of strong well posedness is given in Sects. II and III. We then show in Sect. IV that these results can be applied to electromagnetic and gravitational theory to formulate boundary conditions of practical value for the numerical treatment of isolated systems.

A. Main theorem. Let $T>0$, and denote by $\Sigma$ a $d$-dimensional compact manifold with smooth boundaries $\partial \Sigma$. The type of system our results apply to is a set of quasilinear wave equations on $M=[0, T] \times \Sigma$ coupled both by lower order terms and in the principal part, by a change in the characteristic directions via a metric which can depend on the local value of the fields involved. More precisely, let $\pi: E \rightarrow M$ be a vector bundle

\footnotetext{
1 In theories such as hydrodynamics, the four-velocity determines a preferred time direction and thereby a unique symmetrizer.
} 
over $M$ with fibre $\mathbb{R}^{N}$, let $\nabla_{a}$ be a fixed, given connection on $E$ and let $g_{a b}=g_{a b}(\Phi)$ be a Lorentz metric on $M$ with inverse $g^{a b}(\Phi)$ which depends pointwise and smoothly on a set of fields $\Phi=\left\{\Phi^{A}\right\}_{A=1,2, \ldots N}$ parameterizing a local section of $E$. Our signature convention for $g_{a b}$ is $(-,+, \ldots,+)$. We shall also assume that each time-slice $\Sigma_{t}=\{t\} \times \Sigma$ is space-like and that the boundary $\mathcal{T}=[0, T] \times \partial \Sigma$ is time-like with respect to $g_{a b}(\Phi)$. In the following, we will refer to local sections in $E$ as vector-valued functions over $M$. We will also assume the existence of a positive-definite fibre metric $h_{A B}$ on $E$. We consider a system of quasilinear wave equations of the form

$$
g^{a b}(\Phi) \nabla_{a} \nabla_{b} \Phi^{A}=S^{A}(\Phi, \nabla \Phi),
$$

where $S^{A}(\Phi, \nabla \Phi)$ is a vector-valued function which depends pointwise and smoothly on its arguments. The wave system (1) is subject to the initial conditions

$$
\left.\Phi^{A}\right|_{\Sigma_{0}}=\Phi_{0}^{A},\left.\quad n^{b} \nabla_{b} \Phi^{A}\right|_{\Sigma_{0}}=\Pi_{0}^{A}
$$

where $\Phi_{0}^{A}$ and $\Pi_{0}^{A}$ are given vector-valued functions on $\Sigma_{0}$, and where $n^{b}=n^{b}(\Phi)$ denotes the future-directed unit normal to $\Sigma_{0}$ with respect to $g_{a b}$. In order to describe the boundary conditions, let $T^{a}=T^{a}(p, \Phi)$ be a future-directed vector field which is tangent to $\mathcal{T}$ and which is normalized with respect to $g_{a b}$ and let $N^{a}=N^{a}(p, \Phi)$ be the unit outward normal to $\mathcal{T}$ with respect to the metric $g_{a b}$. We consider boundary conditions on $\mathcal{T}$ of the following form ${ }^{2}$ :

$$
\left.\left[T^{b}+\alpha N^{b}\right] \nabla_{b} \Phi^{A}\right|_{\mathcal{T}}=\left.c^{a A}{ }_{B} \nabla_{a} \Phi^{B}\right|_{\mathcal{T}}+\left.d^{A}{ }_{B} \Phi^{B}\right|_{\mathcal{T}}+G^{A}
$$

where $\alpha=\alpha(p, \Phi)>0$ is a strictly positive, smooth function, $G^{A}=G^{A}(p)$ is a given, vector-valued function on $\mathcal{T}$ and the matrix coefficients $c^{a A}{ }_{B}=c^{a A}{ }_{B}(p, \Phi)$ and $d_{B}^{A}=d^{A}{ }_{B}(p, \Phi)$ are smooth functions of their arguments. Furthermore, we assume that $c^{a A}{ }_{B}$ can be made arbitrarily small in the following sense: Given a local trivialization $\varphi: U \times \mathbb{R}^{N} \mapsto \pi^{-1}(U)$ of $E$ such that $\bar{U} \subset M$ is compact and contains a portion $\mathcal{U}$ of the boundary $\mathcal{T}$, and given $\varepsilon>0$, there exists a smooth map $J: U \rightarrow$ $G L(N, \mathbb{R}), p \mapsto\left(J^{A}{ }_{B}(p)\right)$ such that the transformed matrix coefficients

$$
\tilde{c}^{a A}{ }_{B}:=J_{C}^{A} c^{a C}{ }_{D}\left(J^{-1}\right)_{B}^{D}
$$

satisfy the condition

$$
h_{A B} \tilde{c}^{a A}{ }_{C}(\Phi) \tilde{c}^{b B}{ }_{D}(\Phi) V_{a}{ }^{C} V_{b}{ }^{D} \leq \varepsilon h_{A B} e^{a b}(\Phi) V_{a}{ }^{A} V_{b}{ }^{B},
$$

for all vector-valued one-forms $V_{a}^{A}$ on $\mathcal{U}$, where here and in the following, $e_{a b}$ refers to the Euclidean metric $e_{a b}=g_{a b}+2 T_{a} T_{b}$ which is defined for points on $\mathcal{T}$.

The main result of this paper is:

Theorem 1. The IBVP $(1,2,3)$ is well posed. Given $T>0$ and sufficiently small and smooth initial and boundary data $\Phi_{0}^{A}, \Pi_{0}^{A}$ and $G^{A}$ satisfying the usual compatibility conditions at $\partial \Sigma_{0}$, there exists a unique smooth solution on $M$ satisfying the evolution equation (1), the initial condition (2) and the boundary condition (3). Furthermore, the solution depends continuously on the initial and boundary data.

\footnotetext{
${ }^{2}$ We adopt the Einstein summation convention for the lower case Latin abstract spacetime indices $a, b, c$, $\ldots$ as well as for the Capital indices $A, B, C, \ldots$ on the fibre of $E$.
} 
A common situation in which condition (4) is automatically satisfied is given in the following:

Lemma 1. Let $\mathcal{U} \subset \mathcal{T}$ be an open and bounded subset of $\mathcal{T}$. Assume there exists a smooth map $J: \mathcal{U} \rightarrow G L(N, \mathbb{R}), p \mapsto\left(J^{A}{ }_{B}(p)\right)$ over $\mathcal{U}$ such that the transformed matrix coefficients $\tilde{c}^{a}{ }_{B}:=J^{A}{ }_{C} c^{a C}{ }_{D}\left(J^{-1}\right)^{D}{ }_{B}$ are in upper triangular form with zeroes on the diagonal, that is

$$
\tilde{c}^{a A}=0, \quad B \leq A .
$$

Then, the condition (4) is satisfied on $\mathcal{U}$.

Proof (cf. The proof of the Liapunov stability theorem). In order to simplify the notation we use a matrix notation and write $\tilde{c}^{a}=J c^{a} J^{-1}$. Let $\delta>0$, and define $D_{\delta}:=$ $\operatorname{diag}\left(1, \delta, \delta^{2}, \ldots, \delta^{N-1}\right)$ and $J_{\delta}:=D_{\delta}^{-1} J$. Then, $c_{\delta}^{a}:=J_{\delta} c^{a} J_{\delta}^{-1}=D_{\delta}^{-1} \tilde{c}^{a} D_{\delta}$ has the components $\left(c_{\delta}^{a}\right)^{A}{ }_{B}=\delta^{B-A} \tilde{c}^{a A}{ }_{B}$, where here, $\delta^{B-A}$ refers to the $(B-A)^{\text {th }}$ power of $\delta$. Since $\tilde{c}^{a}{ }_{B}=0$ for $B \leq A$ we have $c_{\delta}^{a}=\mathcal{O}(\delta)$, and $c_{\delta}^{a}$ satisfies condition (4) provided $\delta>0$ is chosen small enough.

The proof of Theorem 1 is given in Sects. II and III. In order to illustrate the ideas on a simpler example, we start in Sect. II with the wave equation on a fixed background metric $g_{a b}$, and analyze the general case in Sect. III.

Since many physical systems can be described by systems of wave equations, Theorem 1 should have many applications. In the following, we mention two such applications for the initial-boundary value formulation of isolated systems with constraints. The physical motivation for the choice of nonreflecting boundary conditions in these examples is described in detail in Sect. IV.

B. Maxwell's equations in the Lorentz gauge. The first application describes an electromagnetic field on the manifold $M=[0, T] \times \Sigma$ with a fixed background metric $g_{a b}$ and corresponding Levi-Civita connection $\nabla_{a}$. As before, we assume that each time-slice $\Sigma_{t}=\{t\} \times \Sigma$ is space-like and that the boundary $\mathcal{T}=[0, T] \times \partial \Sigma$ is time-like. In the Lorentz gauge $C:=\nabla_{b} A^{b}=0$, where $A^{b}$ denotes the 4-vector potential, Maxwell's equations assume the form of a system of wave equations,

$$
g^{a b} \nabla_{a} \nabla_{b} A^{c}=R_{d}^{c} A^{d}-J^{c},
$$

where $R_{a b}$ denotes the Ricci tensor belonging to the metric $g_{a b}$ and $J^{c}$ is the four-current. Equation (5) implies that the constraint variable $C$ obeys the following equation:

$$
g^{a b} \nabla_{a} \nabla_{b} C=-\nabla^{c} J_{c} .
$$

Therefore, the imposition of the boundary condition $\left.C\right|_{\mathcal{T}}=0$ and the satisfaction of the continuity equation $\nabla^{c} J_{c}=0$ imply that any smooth enough solution of (5) with initial data satisfying

$$
\left.C\right|_{\Sigma_{0}}=0,\left.\quad n^{a} \nabla_{a} C\right|_{\Sigma_{0}}=0
$$

satisfies the constraint $C=0$ on $M$ since in this case the constraint propagation system (6) is homogeneous. 
Asymptotically nonreflecting boundary conditions at $\mathcal{T}=[0, T] \times \Sigma$, in the sense of Sect. IV, can be formulated by first introducing a null tetrad $\left\{K^{a}, L^{a}, Q^{a}, \bar{Q}^{a}\right\}$ which is adapted to the boundary. Let $T^{a}$ be a future-directed time-like vector field tangent to $\mathcal{T}$ normalized such that $g_{a b} T^{a} T^{b}=-1$. For example, one can define $T^{a}$ by orthogonal projection of the future-directed normal to the time-slices $\Sigma_{t}$ onto $\mathcal{T}$. Next, let $N^{a}$ denote the unit outward normal to $\mathcal{T}$ with respect to $g_{a b}$ and complete $T^{a}$ and $N^{a}$ to an orthonormal basis $\left\{T^{a}, N^{a}, V^{a}, W^{a}\right\}$ of $T_{p} M$ at each point $p \in \mathcal{T}$. Then, we define the null vectors

$$
K^{a}:=T^{a}+N^{a}, \quad L^{a}:=T^{a}-N^{a}, \quad Q^{a}:=V^{a}+i W^{a}, \quad \bar{Q}^{a}:=V^{a}-i W^{a},
$$

where $i=\sqrt{-1}$. Finally, let $r$ denote the areal radius of the cross sections $\partial \Sigma_{t}$. The following boundary conditions are motivated from the considerations in Sect. IV B:

$$
\begin{gathered}
K^{a} K_{b} \nabla_{a} A^{b}+\left.\frac{2}{r} K_{b} A^{b}\right|_{\mathcal{T}}=q_{K}, \\
\left.\left(K^{a} Q_{b}-Q^{a} K_{b}\right) \nabla_{a} A^{b}\right|_{\mathcal{T}}=q_{Q}, \\
\left.\left(K^{a} L_{b}+L^{a} K_{b}-Q^{a} \bar{Q}_{b}-\bar{Q}^{a} Q_{b}\right) \nabla_{a} A^{b}\right|_{\mathcal{T}}=0,
\end{gathered}
$$

where $q_{K}$ and $q_{Q}$ are given real and complex scalars on $\mathcal{T}$. The first condition is a gauge condition, the second condition controls the electromagnetic radiation through $\mathcal{T}$ and the third condition enforces the constraint $C=g^{a b} \nabla_{a} A_{b}=0$ on $\mathcal{T}$. For the special case of a flat background with a spherical boundary, these boundary conditions reduce to the ones proposed in Sect. IV B which are shown to yield small spurious reflections. Therefore, we expect them to yield small spurious reflections also in the case of asymptotically flat curved spacetimes as long as the boundary is nearly spherical and located far into the wave zone.

The evolution equation (5) has the form (1) where $E$ is the tangent bundle over $M$, and the boundary conditions $(7,8,9)$ have the form $(3)$ with

$$
\begin{aligned}
& \alpha=1, \\
& c^{a c}{ }_{d}=\frac{1}{2}\left[2 Q^{(a} \bar{Q}^{c)} K_{d}+L^{a} K^{c} K_{d}-K^{c}\left(Q^{a} \bar{Q}_{d}+\bar{Q}^{a} Q_{d}\right)\right], \quad d_{d}^{c}=\frac{1}{r} L^{c} K_{d}, \\
& G^{c}=\frac{1}{2}\left[-L^{c} q_{K}+\bar{Q}^{c} q_{Q}+Q^{c} \bar{q}_{Q}\right] .
\end{aligned}
$$

Since

$$
\begin{aligned}
c^{a c}{ }_{d} K^{d} & =0, \\
c^{a c}{ }_{d} Q^{d} & =-Q^{a} K^{c}, \\
c^{a c}{ }_{d} \bar{Q}^{d} & =-\bar{Q}^{a} K^{c}, \\
c^{a c}{ }_{d} L^{d} & =-L^{a} K^{c}-\bar{Q}^{a} Q^{c}-Q^{a} \bar{Q}^{c},
\end{aligned}
$$

the matrix elements $c^{a c}{ }_{d}$ are in upper triangular form with zeroes in the diagonal when expressed in terms of the basis $\left\{K^{a}, Q^{a}, \bar{Q}^{a}, L^{d}\right\}$. Therefore, the assumptions of Lemma 1 are satisfied and we obtain a well posed IBVP. 
C. Einstein's equations in harmonic coordinates. As a second application of our theorem we consider Einstein's field equations in (generalized) harmonic coordinates. For this, we follow $[15,16]$ and choose a fixed background metric $\stackrel{\circ}{g}_{a b}$ on $M=[0, T] \times \Sigma$ with the property that each time-slice $\Sigma_{t}=\{t\} \times \Sigma$ is space-like and the boundary $\mathcal{T}=[0, T] \times \partial \Sigma$ is time-like with respect to $\stackrel{\circ}{g}_{a b}$. We impose the following gauge condition on the dynamical metric $g_{a b}$ :

$$
\mathcal{C}^{c}:=g^{a b}\left(\Gamma_{a b}^{c}-\stackrel{\circ}{\Gamma}_{a b}^{c}\right)-H^{c}=0 .
$$

Here, $H^{c}$ is a given vector field on $M$ and $\Gamma^{c}{ }_{a b}$ and $\stackrel{\circ}{\Gamma}^{c}{ }_{a b}$ are the Christoffel symbols corresponding to the dynamical and background metrics, respectively. In the particular case where $H^{c}=0$ and where the background metric is the Minkowski metric in standard Cartesian coordinates, $\stackrel{\circ}{\Gamma}^{c} a b$ vanishes, and the condition $\mathcal{C}^{c}=0$ reduces to the usual condition for harmonic coordinates $\square x^{\mu}=0$ for $\mu=t, x, y, z$. However, the advantage of the condition (10) is that it maintains the covariance of the theory since $\mathcal{C}^{c}$ is the difference between the two Christoffel symbols,

$$
C_{a b}^{c} \equiv \Gamma_{a b}^{c}-\stackrel{\circ}{\Gamma}_{a b}^{c}=\frac{1}{2} g^{c d}\left(\stackrel{\circ}{\nabla}_{a} h_{b d}+\stackrel{\circ}{\nabla}_{b} h_{a d}-\stackrel{\circ}{\nabla}_{d} h_{a b}\right),
$$

where $h_{a b}=g_{a b}-\stackrel{\circ}{g}_{a b}$ denotes the difference between the dynamical and the background metric.

With the condition (10), Einstein's field equations are equivalent to the wave system

$$
\begin{aligned}
g^{c d} \stackrel{\circ}{\nabla}_{c} \stackrel{\circ}{\nabla}_{d} h_{a b}= & 2 g_{e f} g^{c d} C_{a c}^{e} C_{b d}^{f}+4 C^{c} d\left(a g_{b) e} C^{e}{ }_{c f} g^{d f}-2 g^{c d} \stackrel{\circ}{R}_{c d(a} g_{b) e}\right. \\
& +16 \pi G\left(T_{a b}-\frac{1}{2} g_{a b} g^{c d} T_{c d}\right)+2 \nabla_{(a} H_{b)},
\end{aligned}
$$

where $\stackrel{\circ}{R}_{b c d}{ }_{b c d}$ denotes the curvature tensor with respect to $\stackrel{\circ}{g}_{a b}, T_{a b}$ the stress-energy tensor and $G$ denotes Newton's constant. Solutions of this equation which are smooth enough imply that the constraint variable $\mathcal{C}_{a}$ satisfies

$$
g^{c d} \nabla_{c} \nabla_{d} \mathcal{C}_{a}=-R_{a}{ }^{b} \mathcal{C}_{b}-16 \pi G \nabla^{b} T_{a b}
$$

Therefore, the imposition of the boundary condition $\left.\mathcal{C}_{a}\right|_{\mathcal{T}}=0$ implies that any smooth enough solution of (12) with initial data satisfying

$$
\left.\mathcal{C}_{a}\right|_{\Sigma_{0}}=0,\left.\quad n^{a} \nabla_{a} \mathcal{C}_{b}\right|_{\Sigma_{0}}=0
$$

satisfies the constraint $\mathcal{C}_{a}=0$ on $M$ provided the stress-energy tensor is divergence free, $\nabla^{b} T_{a b}=0$.

In order to formulate asymptotically nonreflecting boundary conditions we first construct an adapted local null tetrad $\left\{K^{a}, L^{a}, Q^{a}, \bar{Q}^{a}\right\}$ as in the electromagnetic case. Notice that here these quantities are defined with respect to the dynamical metric $g_{a b}$ and not the background metric $\stackrel{\circ}{g}_{a b}$ and as a consequence, they depend on $g_{a b}$. However, it is important to note that these vectors do not depend on derivatives of $g_{a b}$. A radial function $r$ on $\mathcal{T}$ is defined as the areal radius of the cross sections $\partial \Sigma_{t}$ with 
respect to the background metric. The boundary conditions which are motivated from the considerations in Sect. IV C are the following:

$$
\begin{gathered}
K^{a} K^{b} K^{c} \stackrel{\circ}{\nabla}_{a} h_{b c}+\left.\frac{2}{r} K^{b} K^{c} h_{b c}\right|_{\mathcal{T}}=-q_{K K}, \\
K^{a} K^{b} L^{c} \stackrel{\circ}{\nabla}_{a} h_{b c}+\frac{1}{r} K^{b} L^{c} h_{b c}+\left.\frac{1}{r} Q^{b} \bar{Q}^{c} h_{b c}\right|_{\mathcal{T}}=-q_{Q \bar{Q}}, \\
K^{a} K^{b} Q^{c} \stackrel{\circ}{\nabla}_{a} h_{b c}+\frac{2}{r} K^{b} Q^{c} h_{b c}=-q_{K}, \\
K^{a} Q^{b} Q^{c} \stackrel{\circ}{\nabla}_{a} h_{b c}-\left.Q^{a} Q^{b} K^{c} \stackrel{\circ}{\nabla}_{a} h_{b c}\right|_{\mathcal{T}}=-q_{Q Q}, \\
\left.\left(K^{a} Q^{b} \bar{Q}^{c}+L^{a} K^{b} K^{c}-Q^{a} K^{b} \bar{Q}^{c}-\bar{Q}^{a} K^{b} Q^{c}\right) \stackrel{\circ}{a}_{a} h_{b c}\right|_{\mathcal{T}}=-\left.2 K^{a} H_{a}\right|_{\mathcal{T}}, \\
\left.\left(K^{a} L^{b} Q^{c}+L^{a} K^{b} Q^{c}-Q^{a} K^{b} L^{c}+\bar{Q}^{a} Q^{b} Q^{c}\right) \stackrel{\circ}{\nabla}_{a} h_{b c}\right|_{\mathcal{T}}=-\left.2 Q^{a} H_{a}\right|_{\mathcal{T}}, \\
\left.\left(K^{a} L^{b} L^{c}+L^{a} Q^{b} \bar{Q}^{c}-Q^{a} \bar{Q}^{b} L^{c}-\bar{Q}^{a} Q^{b} L^{c}\right) \stackrel{\circ}{\nabla}_{a} h_{b c}\right|_{\mathcal{T}}=-\left.2 L^{a} H_{a}\right|_{\mathcal{T}},
\end{gathered}
$$

where $q_{K K}$ and $q_{Q \bar{Q}}$ are real-valued given functions on $\mathcal{T}$ and $q_{K} Q$ and $q_{Q Q}$ are complex-valued given functions on $\mathcal{T}$. The evolution equation (12) has the form (1) where $E$ is the vector bundle of symmetric, covariant tensor fields on $M$ and the boundary conditions (14-20) have the form (3), where $\alpha=1$ and $c^{a b c} d e$ is in upper triangular form when expressed in terms of the basis $\left\{K^{b} K^{c}, K^{(b} L^{c)}, K^{(b} Q^{c)}, Q^{b} Q^{c}, Q^{(b} \bar{Q}^{c)}, L^{(b} Q^{c)}, L^{b} L^{c}\right\}$.

For the case where $\stackrel{\circ}{g}_{a b}$ is the Minkowski metric and $h_{a b}$ is treated as a linear perturbation thereof, the boundary conditions (14-17) reduce to the ones proposed in Sect. IV C for a spherical boundary. As in the preceding application to electrodynamics, we expect these boundary conditions to yield small spurious reflections in the case of a nearly spherical boundary in the wave zone of an asymptotically flat curved spacetime. Their content can be clarified by considering the case of a wave incident on a plane boundary. The discussion in Sect. IV A shows that the first three conditions (14),(15) and (16) are related to the gauge freedom; and the condition (17) controls the gravitational radiation. The remaining conditions (18),(19) and (20) enforce the constraint $\mathcal{C}_{a}=0$ on the boundary.

\section{The Wave Equation on a Curved Background}

In this section we prove Theorem 1 for the case of a single wave equation

$$
g^{a b} \nabla_{a} \nabla_{b} \phi=S
$$

on $M=[0, T] \times \Sigma$. For simplicity, we also assume that $g_{a b}$ and $S$ are independent of $\phi$ and that $\nabla_{a}$ is the Levi-Civita connection with respect to $g_{a b}$. The IBVP consists in finding solutions of (21) subject to the initial conditions

$$
\left.\phi\right|_{\Sigma_{0}}=\phi_{0},\left.\quad n^{b} \nabla_{b} \phi\right|_{\Sigma_{0}}=\pi_{0}
$$

where $\phi_{0}$ and $\pi_{0}$ are given functions on $\Sigma_{0}$, and the boundary conditions

$$
\left[T^{b} \nabla_{b} \phi+\alpha N^{b} \nabla_{b} \phi\right]_{\mathcal{T}}=G
$$


where $G$ is a given function on $\mathcal{T}$. Here, $n^{b}$ and $N^{b}$ denote the future-directed unit vector field to the time-slices $\Sigma_{t}$ and the outward unit normal vector field to $\mathcal{T}$, respectively, $T^{b}$ is an arbitrary future-directed time-like vector field which is tangent to the boundary surface $\mathcal{T}$ and $\alpha$ is a strictly positive function on $\mathcal{T}$. Without loss of generality, we assume that $T^{a}$ is normalized such that $g_{a b} T^{a} T^{b}=-1$. Furthermore, by redefining $\phi$ and $S$ if necessary, we may also assume that the boundary data $G$ vanishes identically.

In order to show well posedness for this problem, we use a geometric reduction to a first order symmetric hyperbolic system with maximal dissipative boundary conditions $[3,4,17]$. First, introducing the variables $V_{a}=\nabla_{a} \phi$, the wave equation can be rewritten as the first order system

$$
\begin{gathered}
\nabla_{a} \phi=V_{a}, \\
g^{a b} \nabla_{a} V_{b}=S, \\
\nabla_{a} V_{b}-\nabla_{b} V_{a}=0 .
\end{gathered}
$$

Next, we specify any future-directed time-like vector field $u^{a}$ and contract the first and the last equation with it. This yields the evolution system

$$
\begin{gathered}
£_{u} \phi=u^{a} V_{a} \equiv \Pi, \\
g^{a b} \nabla_{a} V_{b}=S, \\
£_{u} V_{b}=\nabla_{b} \Pi,
\end{gathered}
$$

where $f_{u}$ denotes the Lie derivative with respect to $u^{a}$. This system is subject to the initial and boundary conditions

$$
\begin{gathered}
\left.\phi\right|_{\Sigma_{0}}=\phi_{0},\left.\quad n^{b} V_{b}\right|_{\Sigma_{0}}=\pi_{0}, \quad \iota_{0}^{*} V_{b}=\iota_{0}^{*} \nabla_{b} \phi_{0}, \\
{\left[T^{b} V_{b}+\alpha N^{b} V_{b}\right]_{\mathcal{T}}=0,}
\end{gathered}
$$

where $\iota_{0}: \Sigma_{0} \rightarrow M$ is the inclusion map, and subject to the constraint $C_{a}=0$, where the constraint variable $C_{a}$ is defined as $C_{a}=V_{a}-\nabla_{a} \phi$. The evolution equations (27) and (29) imply that $C_{a}$ is Lie-dragged by the time evolution vector field $u^{a}$,

$$
£_{u} C_{a}=0 .
$$

In the following, we assume that $u^{a}$ is pointing away from the domain at the boundary. This implies that a solution of $(27,28,29)$ with constraint-satisfying initial data automatically satisfies the constraints everywhere on $M$, and no extra boundary conditions are needed in order to ensure that the constraint $C_{a}=0$ propagates.

Still, there is a huge freedom in choosing the evolution vector field $u^{a}$; different choices lead to first order evolution systems $(27,28,29)$ which are inequivalent to each other if the solution is off the constraint surface $C_{a}=0$. In this work we exploit this freedom in order to obtain energy estimates which allow for an appropriate control of the fields not only in the bulk but also on the boundary of the domain (see the estimate (37) below). In order to analyze this, following [17] we rewrite the evolution system $(28,29)$ in the form

$$
\mathcal{A}^{a}{ }_{b c} \nabla_{a} V^{c} \equiv-u^{a}\left(\nabla_{a} V_{b}-\nabla_{b} V_{a}\right)+u_{b} \nabla_{a} V^{a}=u_{b} S,
$$


where the symbol is given by $\mathcal{A}^{a} b c=-u^{a} g_{b c}+2 \delta^{a}{ }_{(b} u_{c)}$. Since $\mathcal{A}^{a}{ }_{b c}$ is symmetric in $b c$ and since $u_{a} \mathcal{A}^{a} b c=-u_{a} u^{a} g_{b c}+2 u_{b} u_{c}$ is positive definite, the evolution system is symmetric hyperbolic. In particular, the evolution equations imply that

$$
\nabla_{a}\left(\mathcal{A}_{b c}^{a} V^{b} V^{c}\right)=\left(\nabla_{a} \mathcal{A}_{b c}^{a}\right) V^{b} V^{c}+2\left(u_{b} V^{b}\right) S .
$$

Integrating both sides of this equation over the manifold $M=[0, T] \times \Sigma$ and using Gauss' theorem, one obtains ${ }^{3}$

$$
\begin{aligned}
\int_{\Sigma_{T}} n_{a} \mathcal{A}^{a}{ }_{b c} V^{b} V^{c}= & \int_{\Sigma_{0}} n_{a} \mathcal{A}^{a}{ }_{b c} V^{b} V^{c}+\int_{\mathcal{T}} N_{a} \mathcal{A}^{a}{ }_{b c} V^{b} V^{c} \\
& -\int_{M}\left[\left(\nabla_{a} \mathcal{A}^{a}{ }_{b c}\right) V^{b} V^{c}+2\left(u_{b} V^{b}\right) S\right] .
\end{aligned}
$$

The following two conditions from the theory of symmetric linear operators (see [4]) guarantee that the first order IBVP $(27,28,29,30,31)$ is well posed:

(i) $n_{a} \mathcal{A}^{a}{ }_{b c}$ is positive definite.

(ii) For each $p \in \mathcal{T}$, the subspace $\mathcal{N}_{-}(p) \subset T_{p} M$ consisting of the vectors $V^{b}(p)$ satisfying the boundary condition (31) at $p$ is maximal non-positive. This means that $N_{a} \mathcal{A}^{a}{ }_{b c}(p) V^{b}(p) V^{c}(p) \leq 0$ for all $V^{b}(p) \in \mathcal{N}_{-}(p)$ and that $\mathcal{N}_{-}(p)$ does not possess a proper extension with this property.

For the following, we choose the time evolution vector field $u^{a}$ such that $u^{a}$ is everywhere future-directed and time-like on $M$ and such that $u^{a}$ lies in the plane spanned by $T^{a}$ and $N^{a}$ at each point of the boundary, more specifically,

$$
\left.u^{a}\right|_{\mathcal{T}}=T^{a}+\delta N^{a},
$$

with $0<\delta<1$ a function on $\mathcal{T}$. The following two lemmas imply the satisfaction of the conditions (i) and (ii) for an appropriate choice of $\delta$.

Lemma 2. $n_{a} \mathcal{A}_{b c}^{a}(p)$ is positive definite for all $p \in M$.

Proof Let $h_{a b}=g_{a b}+n_{a} n_{b}$ be the induced metric on $\Sigma_{t}$ and expand $u_{a}=\mu\left(n_{a}+\bar{u}_{a}\right)$, where $\mu=-n^{a} u_{a}$. Since $u^{a}$ is future-directed and time-like, $\mu>0$ and $\bar{u}^{a} \bar{u}_{a}<1$. Therefore,

$$
n_{a} \mathcal{A}_{b c}^{a}=\mu\left(h_{b c}+n_{b} n_{c}+2 n_{(b} \bar{u}_{c)}\right)
$$

is positive definite.

Lemma 3. Let $0<\delta \leq \alpha\left(1+\alpha^{2}\right)^{-1}$. Then, the boundary spaces $\mathcal{N}_{-}(p)$ are maximal non-positive for all $p \in \mathcal{T}$.

3 Notice that since $n^{a}$ is future directed, its flow increases $t$; hence in coordinates $\left(t, x^{i}\right)$, where $t$ parametrizes $[0, T]$ and $x^{i}$ are local coordinates on $\Sigma$, we have $n^{t}>0$ and $n_{t}<0$. 
Proof (cf. Appendix B in Ref. [9]). Fix a point $p \in \mathcal{T}$, and let $V^{b} \in T_{p} M$. We have

$$
\begin{aligned}
N_{a} \mathcal{A}^{a}{ }_{b c} V^{b} V^{c}= & {\left[\delta T_{b} T_{c}+\delta N_{b} N_{c}+2 T_{(b} N_{c)}-\delta H_{b c}\right] V^{b} V^{c} } \\
= & -\delta\left[\left(T^{b} V_{b}\right)^{2}+\left(N^{b} V_{b}\right)^{2}+H_{b c} V^{b} V^{c}\right] \\
& +2\left[\delta\left(T^{b} V_{b}\right)^{2}+\delta\left(N^{b} V_{b}\right)^{2}+\left(T^{b} V_{b}\right)\left(N^{c} V_{c}\right)\right],
\end{aligned}
$$

where $H_{b c}=g_{b c}+T_{b} T_{c}-N_{b} N_{c}$ is the induced metric on the orthogonal complement of the plane spanned by $T^{b}$ and $N^{b}$. Eliminating the terms $\left(T^{b} V_{b}\right)$ in the second square bracket on the right-hand side using the boundary condition (31) we obtain

$$
N_{a} \mathcal{A}_{b c}^{a} V^{b} V^{c}=-\delta\left[\left(T^{b} V_{b}\right)^{2}+\left(N^{b} V_{b}\right)^{2}+H_{b c} V^{b} V^{c}\right]+2\left[\delta\left(\alpha^{2}+1\right)-\alpha\right]\left(N^{b} V_{b}\right)^{2} .
$$

The last term on the right-hand side is non-positive by the assumption of the lemma. Therefore, $N_{a} \mathcal{A}^{a}{ }_{b c}$ is negative-definite on the subspace of vectors $V^{a}$ satisfying the boundary condition. Finally, we observe that $\mathcal{N}_{-}(p)$ is maximal since its dimension is $d=\operatorname{dim} T_{p} M-1$, while the symmetric bilinear form $N_{a} \mathcal{A}^{a}{ }_{b c}$ has signature $(1, d)$.

If we relax the assumption of homogeneous boundary data and replace the condition (31) by the condition

$$
\left[T^{b} V_{b}+\alpha N^{b} V_{b}\right]_{\mathcal{T}}=G
$$

we obtain, instead of (33),

$$
\begin{aligned}
N_{a} \mathcal{A}^{a}{ }_{b c} V^{b} V^{c}= & -\delta\left[\left(T^{b} V_{b}\right)^{2}+\left(N^{b} V_{b}\right)^{2}+H_{b c} V^{b} V^{c}\right]+2\left[\delta\left(\alpha^{2}+1\right)-\alpha\right]\left(N^{b} V_{b}\right)^{2} \\
& +2(1-2 \delta \alpha)\left(N^{b} V_{b}\right) G+2 \delta G^{2} .
\end{aligned}
$$

Let $0<\rho<1$ and set $\delta=(1-\rho) \alpha\left(1+\alpha^{2}\right)^{-1}$. Then, we have (cf. Appendix B in Ref. [9])

$$
N_{a} \mathcal{A}_{b c}^{a} V^{b} V^{c} \leq-\delta\left[\left(T^{b} V_{b}\right)^{2}+\left(N^{b} V_{b}\right)^{2}+H_{b c} V^{b} V^{c}\right]+\left[2 \delta+\frac{(1-2 \delta \alpha)^{2}}{2 \alpha \rho}\right] G^{2}
$$

This and the positivity of $n_{a} \mathcal{A}_{b c}^{a}$ implies the existence of strictly positive constants $C_{1}$ and $C_{2}$ (depending on $\delta$ and $\rho$ ) such that

$$
N_{a} \mathcal{A}^{a}{ }_{b c} V^{b} V^{c} \leq-C_{1} n_{a} \mathcal{A}^{a}{ }_{b c} V^{b} V^{c}+C_{2} G^{2} .
$$

Using this in the identity (32) we obtain the estimate

$$
\begin{aligned}
\int_{\Sigma_{t}} n_{a} \mathcal{A}^{a}{ }_{b c} V^{b} V^{c} \leq & \int_{\Sigma_{0}} n_{a} \mathcal{A}^{a}{ }_{b c} V^{b} V^{c}-C_{1} \int_{\mathcal{T}_{t}} n_{a} \mathcal{A}^{a}{ }_{b c} V^{b} V^{c}+C_{2} \int_{\mathcal{T}_{t}} G^{2} \\
& +C_{3} \int_{0}^{t}\left[\int_{\Sigma_{s}} n_{a} \mathcal{A}^{a}{ }_{b c} V^{b} V^{c}+\int_{\Sigma_{s}} S^{2}\right] d s
\end{aligned}
$$


for all $0 \leq t \leq T$, where $C_{1}, C_{2}$ and $C_{3}$ are strictly positive constants which are independent of $V^{\bar{b}}$, and $\mathcal{T}_{t}:=[0, t] \times \partial \Sigma$. Applying Gronwall's lemma ${ }^{4}$ to the function $y(t):=\int_{0}^{t} \int_{\Sigma_{s}} n_{a} \mathcal{A}^{a} b c V^{b} V^{c} d s$ we obtain from this

Lemma 4. Let $T>0$. There is a constant $C=C(T) \geq 1$ such that all smooth enough solutions to the IBVP $(28,29,30,34)$ satisfy the inequality

$$
\begin{aligned}
& \int_{\Sigma_{t}} n_{a} \mathcal{A}^{a}{ }_{b c} V^{b} V^{c}+\int_{\mathcal{T}_{t}} n_{a} \mathcal{A}^{a}{ }_{b c} V^{b} V^{c} \\
& \leq C\left[\int_{\Sigma_{0}} n_{a} \mathcal{A}^{a}{ }_{b c} V^{b} V^{c}+\int_{\mathcal{T}_{t}} G^{2}+\int_{0}^{t}\left(\int_{\Sigma_{s}} S^{2}\right) d s\right],
\end{aligned}
$$

for all $0 \leq t \leq T$, where $\mathcal{T}_{t}:=[0, t] \times \partial \Sigma$.

Since any solution of this problem also satisfies $u^{a} C_{a}=u^{a} V_{a}-£_{u} \phi=0, £_{u} C_{a}=0$ and $\iota_{0}^{*} C_{a}=\iota_{0}^{*}\left(V_{a}-\nabla_{a} \phi\right)=0$, and since $u^{a}$ points outward from the domain at $\mathcal{T}$, the constraint $C_{a}=0$ is satisfied everywhere on $M$. From this and the previous lemma, we have established:

Theorem 2. The second order problem $(21,22,23)$ is strongly well posed: given smooth initial and boundary data $\phi_{0}, \pi_{0}$ and $G$ satisfying the usual compatibility conditions at $\partial \Sigma_{0}$, there exists a unique smooth solution satisfying the estimate (37) with $V^{a}$ replaced by $\nabla^{a} \phi$.

Remark 1. The important feature of the estimate (37) is the second term on the lefthand side which yields a $L^{2}$ boundary estimate for the gradient of $\phi$. This estimate is obtained by choosing the time evolution vector field $u^{a}$ in such a way that the boundary matrix $N_{a} \mathcal{A}_{b c}^{a}$ is negative definite on the subspace of vectors satisfying the boundary conditions. As we will see (Lemma 6 in the next section), this property is important for systems of wave equations since it allows the coupling of the boundary conditions through small enough terms involving first derivatives of the fields. If, on the other hand, $u^{a}$ is chosen to be tangent to the boundary, the boundary matrix has a nontrivial kernel and one does not obtain an estimate for the full gradient of $\phi$ on the boundary from the first order system. However, this does not affect the strong well posedness of the second order system which is independent of $u^{a}$.

As an example, consider the wave equation on the half-plane $\Sigma=\mathbb{R}_{+} \times \mathbb{R}^{2}$ with the flat metric $g=-d t^{2}+d x^{2}+d y^{2}+d z^{2}$. In this case, we have

$$
n^{a} \partial_{a}=\partial_{t}, \quad N^{a} \partial_{a}=-\partial_{x} \quad T^{a} \partial_{a}=\frac{1}{p}\left(\partial_{t}-\beta^{y} \partial_{y}-\beta^{z} \partial_{z}\right)
$$

with $\left(\beta^{y}\right)^{2}+\left(\beta^{z}\right)^{2}<1$ and $p:=\sqrt{1-\left(\beta^{y}\right)^{2}-\left(\beta^{z}\right)^{2}}$, and the boundary condition (23) reduces to

$$
\left[\phi_{t}+p \alpha \phi_{x}-\beta^{y} \phi_{y}-\beta^{z} \phi_{z}\right]_{x=0}=p G,
$$

\footnotetext{
4 See, for instance, Lemma 3.1.1 in Ref. [18].
} 
where $\phi_{t}:=\partial_{t} \phi$ etc. Choosing $u^{a}=p\left(T^{a}+\delta N^{a}\right)$ with $0<\delta<1$, the energy norm for this problem reads

$\int_{\Sigma_{t}} n_{a} \mathcal{A}_{b c}^{a} V^{b} V^{c}=\int_{0}^{\infty} \int_{-\infty}^{\infty} \int_{-\infty}^{\infty}\left[\phi_{t}^{2}+\phi_{x}^{2}+\phi_{y}^{2}+\phi_{z}^{2}+2 \phi_{t}\left(\delta p \phi_{x}+\beta^{y} \phi_{y}+\beta^{z} \phi_{z}\right)\right] d y d z d x$.

This is similar to the norm we used in Ref. [9] for obtaining an a priori energy estimate for the second order wave equation with boundary condition (38).

\section{Systems of Wave Equations and Proof of Main Theorem}

In order to show that the system $(1,2,3)$ yields a well posed IBVP, we follow the arguments given in Sect. II and reduce it to a first order symmetric hyperbolic system with maximal dissipative boundary conditions. Let $V_{a}{ }^{A}:=\nabla_{a} \Phi^{A}$, and let $u^{a}(p, \Phi)$ denote a future-directed time-like vector field on $M$ such that

$$
\left.u^{a}\right|_{\mathcal{T}}=T^{a}+\delta N^{a},
$$

with $0<\delta<1$ a function on $\mathcal{T}$ to be determined. Then (1) can be rewritten as the first order evolution system

$$
\begin{gathered}
u^{a} \nabla_{a} \Phi^{A}=u^{a} V_{a}{ }^{A}, \\
g^{a b}(\Phi) \nabla_{a} V_{b}{ }^{A}=S^{A}(\Phi, V), \\
u^{a}\left(\nabla_{a} V_{b}{ }^{A}-\nabla_{b} V_{a}{ }^{A}\right)=u^{a} R^{A}{ }_{B a b} \Phi^{B},
\end{gathered}
$$

where $R_{B a b}^{A}$ denotes the curvature belonging to the connection $\nabla_{a}$. At this point, we stress that the connection $\nabla_{a}$ is a fixed background connection on the vector bundle $E$, and not the Levi-Civita connection belonging to the metric $g_{a b}(\Phi)$, so that $R_{B a b}^{A}$ does not depend on $\Phi$ nor its derivatives. The system $(39,40,41)$ is subject to the constraint $C_{b}{ }^{A}=0$, where $C_{b}{ }^{A}:=\nabla_{b} \Phi^{A}-V_{b}{ }^{A}$. Equations $(39,41)$ imply that the constraint variable $C_{b}{ }^{A}$ is Lie-dragged by $u^{a}$ :

$$
\mathfrak{£}_{u}{C_{b}}^{A} \equiv u^{a} \nabla_{a} C_{b}{ }^{A}+\left(\nabla_{b} u^{a}\right) C_{a}{ }^{A}=0 .
$$

Therefore, any smooth enough solution of the first order problem $(39,40,41)$ belonging to initial data with $C_{b}{ }^{A}=0$ satisfies the constraint $C_{b}{ }^{A}=0$ everywhere it is defined. The initial condition is

$$
\left.\Phi^{A}\right|_{\Sigma_{0}}=\Phi_{0}^{A},\left.\quad n^{b} V_{b}^{A}\right|_{\Sigma_{0}}=\Pi_{0}^{A}, \quad \iota_{0}^{*} V_{b}^{A}=\iota_{0}^{*} \nabla_{b} \Phi_{0}^{A},
$$

and the boundary condition (3) reads

$$
\left[T^{b} V_{b}+\alpha N^{b} V_{b}\right]_{\mathcal{T}}=\left.c^{a A}{ }_{B} V_{a}{ }^{B}\right|_{\mathcal{T}}+\left.d^{A}{ }_{B} \Phi^{B}\right|_{\mathcal{T}}+G^{A}
$$

In order to analyze the well posedness of the first order IBVP $(39,40,41,42,43)$ we first linearize the system by replacing the coefficients $g_{a b}(\Phi), S^{A}(\Phi, \nabla \Phi), T^{b}(\Phi)$, $N^{b}(\Phi), \alpha(\Phi), c^{a A_{B}}(\Phi), d^{A}{ }_{B}(\Phi)$ by smooth functions $g_{a b}, S^{A}, T^{b}, N^{b}, \alpha, c^{a A}{ }_{B}, d^{A}{ }_{B}$, respectively. Local in time well posedness for the original quasilinear system follows 
by iteration from the well posedness result for the linear system with enough differentiability ${ }^{5}$. Next, we use a partition of unity in order to localize the problem. With this, it is sufficient to consider a local trivialization $\varphi: U \times \mathbb{R}^{N} \mapsto \pi^{-1}(U)$ of $E$ such that $\bar{U} \subset M$ is compact and contains a portion $\mathcal{U}$ of the boundary $\mathcal{T}$. Let $\varepsilon>0$. According to the assumption there exists a smooth map $J_{\varepsilon}: U \rightarrow G L(N, \mathbb{R}), p \mapsto\left(J_{\varepsilon}(p)\right)$ such that the transformed matrix coefficients $\tilde{c}^{a}:=J_{\varepsilon} c^{a} J_{\varepsilon}^{-1}$ satisfy the condition (4) for all vector-valued one-forms $V_{a}$ on $\mathcal{U}$. Setting $h_{A B}(\varepsilon):=\left(J_{\varepsilon}^{T} h J_{\varepsilon}\right)_{A B}=h_{C D}\left(J_{\varepsilon}\right)^{C}{ }_{A}\left(J_{\varepsilon}\right)^{D}{ }_{B}$, we can reformulate this condition by stating that

$$
h_{A B}(\varepsilon) c^{a A}{ }_{C}(\Phi) c^{b B}{ }_{D}(\Phi) V_{a}{ }^{C} V_{b}{ }^{D} \leq \varepsilon h_{A B}(\varepsilon) e^{a b}(\Phi) V_{a}^{A} V_{b}{ }^{B},
$$

for all vector-valued one-forms $V_{a}^{A}$ on $\mathcal{U}$. The system $(39,40,41)$ can be written in the form

$$
\left(\begin{array}{cc}
-\Lambda h_{A B}(\varepsilon) u^{a} \nabla_{a} & 0 \\
0 & h_{A B}(\varepsilon) \mathcal{A}^{a}{ }_{b c} \nabla_{a}
\end{array}\right)\left(\begin{array}{c}
\Phi^{B} \\
V^{c B}
\end{array}\right)=\mathcal{S}(\Phi, V),
$$

where $\Lambda>0$ is to be determined, $\mathcal{A}^{a}{ }_{b c}=-u^{a} g_{b c}+2 \delta^{a}{ }_{(b} u_{c)}$ and

$$
\mathcal{S}(\Phi, V)=\left(\begin{array}{l}
-\Lambda h_{A B}(\varepsilon) u^{a} V_{a}{ }^{B} \\
-h_{A B}(\varepsilon) R^{B}{ }_{C a b} \Phi^{C} u^{a}+h_{A B}(\varepsilon) u_{b} S^{B}(\Phi, V)
\end{array}\right) .
$$

Let $B\left(n_{a} ;(\Psi, W),(\Phi, V)\right)$ denote the bilinear form belonging to the principal symbol of (45), that is, for an arbitrary one-form $w_{a}$ on $M$ define

$$
B\left(w_{a} ;(\Psi, W),(\Phi, V)\right):=-\Lambda u^{a} w_{a} h_{A B}(\varepsilon) \Psi^{A} \Phi^{B}+h_{A B}(\varepsilon) w_{a} \mathcal{A}_{b c}^{a} W^{b A} V^{c B} .
$$

We have

Lemma 5. Let $\Lambda>0$. Then, $B\left(n_{a} ;(\Psi, W),(\Phi, V)\right)$ is symmetric in $(\Psi, W),(\Phi, V)$ and positive definite for $w_{a}=u_{a}$ and $w_{a}=n_{a}$. Therefore, the system (45) is symmetric hyperbolic.

Proof The symmetry property follows immediately from the symmetry of $h_{A B}(\varepsilon)$ and the symmetry of $\mathcal{A}^{a}{ }_{b c}$ in $b c$. In order to check the positivity statements, let $w_{a}=u_{a}$, $\gamma:=\sqrt{-u^{a} u_{a}}$ and $\hat{u}_{a}:=\gamma^{-1} u_{a}$. Since $\mathcal{A}^{a}{ }_{b c} u_{a}=\gamma^{2}\left[g_{b c}+2 \hat{u}_{b} \hat{u}_{c}\right]$, we find

$$
B\left(u_{a} ;(\Phi, V),(\Phi, V)\right)=\gamma^{2}\left[\Lambda h_{A B}(\varepsilon) \Phi^{A} \Phi^{B}+\left(g_{a b}+2 \hat{u}_{a} \hat{u}_{b}\right) h_{A B}(\varepsilon) V_{a A} V^{b B}\right]
$$

which is manifestly positive definite. The proof that $B\left(n_{a} ;(\Phi, V),(\Phi, V)\right)$ is positive definite is similar to the proof of Lemma 2.

As in the previous section we obtain well posedness of the linearized system provided we can show that each boundary space

$$
\begin{aligned}
\mathcal{N}_{-}(p) & :=\left\{(\Phi, V) \in \mathbb{R}^{N} \times \mathbb{R}^{(d+1) N}:\left[T^{b}(p)+\alpha(p) N^{b}(p)\right] V_{b}{ }^{A}\right. \\
& \left.=c^{a A}{ }_{B}(p) V_{a}{ }^{B}+d^{A}{ }_{B}(p) \Phi^{B}\right\}, \quad p \in \mathcal{U},
\end{aligned}
$$

is maximal non-positive with respect to $B\left(N_{a} ;(\Phi, V),(\Phi, V)\right)$. This is the statement of the next lemma.

\footnotetext{
${ }^{5}$ See, for instance, $[10,18]$.
} 
Lemma 6. Set $\delta:=\alpha\left(1+\alpha^{2}\right)^{-1} / 2$ and $\kappa:=2\left[2 \delta+(1-2 \delta \alpha)^{2} / \alpha\right]^{2}$. Choose $\varepsilon>0$ small enough such that $\kappa \varepsilon<\delta$ and $\Lambda>0$ large enough such that $2 \kappa h_{A B}(\varepsilon) d^{A}{ }_{C} d^{B}{ }_{D} \Phi^{C} \Phi^{D} \leq$ $\delta \Lambda h_{A B}(\varepsilon) \Phi^{A} \Phi^{B}$ for all $\Phi \in \mathbb{R}^{N}$. Then, the boundary space $\mathcal{N}_{-}(p)$ is maximal nonpositive for all $p \in \mathcal{U}$.

Proof Let $p \in \mathcal{U}$. We have, as in the proof of Lemma 3,

$$
\begin{aligned}
B\left(N_{a} ;(\Phi, V),(\Phi, V)\right)= & -\Lambda u^{a} N_{a} h_{A B}(\varepsilon) \Phi^{A} \Phi^{B}+h_{A B}(\varepsilon) N_{a} \mathcal{A}^{a}{ }_{b c} V^{b A} V^{c B} \\
= & -\delta h_{A B}(\varepsilon)\left[\left(T^{a} T^{b}+N^{a} N^{b}+H^{a b}\right) V_{a}{ }^{A} V_{b}{ }^{B}+\Lambda \Phi^{A} \Phi^{B}\right] \\
& +2\left[\delta T^{a} T^{b}+\delta N^{a} N^{b}+T^{a} N^{b}\right] h_{A B}(\varepsilon) V_{a}{ }^{A} V_{b}{ }^{B} .
\end{aligned}
$$

Let $\left(\Phi^{A}, V_{a}{ }^{A}\right) \in \mathcal{N}(p)$. Then, $T^{a} V_{a}{ }^{A}=-\alpha N^{a} V_{a}{ }^{A}+\tilde{G}^{A}$ with $\tilde{G}^{A}:=c^{a}{ }_{B} V_{a}{ }^{B}+$ $d^{A}{ }_{B} \Phi^{B}$, and we may use this equation in order to eliminate the terms $\left(T^{a} V_{a}{ }^{A}\right)$ in the second bracket on the right-hand side of (46). This yields

$$
\begin{aligned}
B\left(N_{a} ;(\Phi, V),(\Phi, V)\right) \leq & -\delta h_{A B}(\varepsilon)\left[\left(T^{a} T^{b}+N^{a} N^{b}+H^{a b}\right) V_{a}{ }^{A} V_{b}{ }^{B}+\Lambda \Phi^{A} \Phi^{B}\right] \\
& +\left[2 \delta+\frac{(1-2 \delta \alpha)^{2}}{\alpha}\right] h_{A B}(\varepsilon) \tilde{G}^{A} \tilde{G}^{B},
\end{aligned}
$$

where we have set $\delta:=\alpha\left(1+\alpha^{2}\right)^{-1} / 2$ and used the boundary estimate (35) with $\rho=1 / 2$. Now,

$$
\begin{aligned}
h_{A B}(\varepsilon) \tilde{G}^{A} \tilde{G}^{B} & \leq 2 h_{A B}(\varepsilon) c^{a A}{ }_{C} V_{a}{ }^{C} c^{b B}{ }_{D} V_{b}{ }^{D}+2 h_{A B}(\varepsilon) d^{A}{ }_{C} \Phi^{C} d^{B}{ }_{D} \Phi^{D} \\
& \leq 2 \varepsilon h_{A B}(\varepsilon) e^{a b} V_{a}{ }^{A} V_{b}{ }^{B}+2 h_{A B}(\varepsilon) d^{A}{ }_{C} d^{B}{ }_{D} \Phi^{C} \Phi^{D},
\end{aligned}
$$

where we have used the estimate (44) in the last step. Recalling that $e^{a b}=g^{a b}+2 T^{a} T^{b}=$ $T^{a} T^{b}+N^{a} N^{b}+H^{a b}$ and the definition of $\kappa$ in the assumption of the lemma we find

$$
\begin{aligned}
B\left(N_{a} ;(\Phi, V),(\Phi, V)\right) \leq & -\delta h_{A B}(\varepsilon)\left[e^{a b} V_{a}{ }^{A} V_{b}{ }^{B}+\Lambda \Phi^{A} \Phi^{B}\right] \\
& +\kappa\left[\varepsilon h_{A B}(\varepsilon) e^{a b} V_{a}{ }^{A} V_{b}{ }^{B}+h_{A B}(\varepsilon) d^{A}{ }_{C} d^{B}{ }_{D} \Phi^{C} \Phi^{D}\right] .
\end{aligned}
$$

The non-positivity of $\mathcal{N}_{-}(p)$ now follows from the assumptions on $\varepsilon$ and $\Lambda$. Finally, we observe that an element in $\mathcal{N}_{-}(p)$ is characterized by $N$ linear conditions in a $(d+2) N-$ dimensional vector space which implies that $\operatorname{dim} \mathcal{N}_{-}(p) \geq(d+1) N$. On the other hand, from Eq. (46) we see that the signature of $B\left(N_{a} ; .,.\right)$ is given by $(N,(d+1) N)$. Therefore, $\operatorname{dim} \mathcal{N}_{-}(p)=(d+1) N$ and the maximality of $\mathcal{N}_{-}(p)$ follows.

\section{Boundary Conditions for Isolated Systems}

We consider here boundary conditions for an isolated system emitting radiation. If, for computational purposes, the evolution domain of such a system has a finite (artificial) boundary, some artificial boundary condition must be imposed. If one knew the correct boundary data for the analytic problem, then in principle one could use any boundary condition corresponding to a well posed IBVP. However, the determination of the correct boundary data is in general a global problem, in which the boundary data must be determined by extending the solution to infinity either by matching to an exterior (linearized or nonlinear) solution obtained by some other means. The matching approach 
has been reviewed elsewhere [19]. Here we consider an alternative approach in which homogeneous boundary data can be assigned in such a way that the accuracy of the boundary condition becomes exact in the limit that the boundary is extended to infinity. (Such boundary conditions would also be beneficial to the matching approach because the corresponding boundary data would be small so that numerical or other error would also have a small effect.) Artificial boundary conditions for an isolated radiating system for which homogeneous data is approximately valid are commonly called absorbing boundary conditions (see e.g. [20-25]), or nonreflecting boundary conditions (see e.g. [26-28]) or radiation boundary conditions (see e.g. [29]). Such boundary conditions are advantageous for computational use. However, local artificial boundary conditions are not perfectly nonreflecting in general. Here, to be more precise, we consider nonreflecting boundary conditions in the sense of boundary conditions for a well posed problem for which homogeneous data produces no spurious reflection in the limit that the boundary approaches an infinite sphere. The extensive literature on improved versions of nonreflecting boundary conditions involves higher order and nonlocal methods. Our interest here is to investigate the optimal choice of local first order homogeneous boundary conditions on a spherical boundary for the constrained Maxwell and linearized Einstein problems expressed in terms of the gauge dependent variables $A^{\mu}$ and $\gamma^{\mu \nu}$. See [30-32] for the construction of higher-order and higher-accurate boundary conditions for Einstein's equations.

We base our discussion on waves from an isolated system satisfying a system of flat space wave equations. We use Greek indices to denote standard inertial coordinates $x^{\mu}=(t, x, y, z)$ in which the components of the Minkowski metric $\eta^{\mu \nu}$ are $\operatorname{diag}(-1,1,1,1)$. In the case of a scalar field $\Phi$, we thus consider the wave equation

$$
\eta^{\alpha \beta} \partial_{\alpha} \partial_{\beta} \Phi=\left(-\partial_{t}^{2}+\partial_{x}^{2}+\partial_{y}^{2}+\partial_{z}^{2}\right) \Phi=S,
$$

where the source $S$ has compact support. Outside the source, we assume that the solution has the form

$$
\Phi=\frac{f(t-r, \theta, \phi)}{r}+\frac{g(t-r, \theta, \phi)}{r^{2}}+\frac{h(t, r, \theta, \phi)}{r^{3}},
$$

where $(r, \theta, \phi)$ are standard spherical coordinates and $f, g$ and $h$ and their derivatives are smooth bounded functions. These assumptions determine the exterior retarded field of a system emitting outgoing radiation. The simplest case is the monopole radiation

$$
\Phi=\frac{f(t-r)}{r}
$$

which satisfies $\left(\partial_{t}+\partial_{r}\right)(r \Phi)=0$. This motivates the use of a Sommerfeld condition

$$
\left.\frac{1}{r}\left(\partial_{t}+\partial_{r}\right)(r \Phi)\right|_{R}=q(t, R, \theta, \phi)
$$

on a finite boundary $r=R$.

The resulting Sommerfeld boundary data $q$ in the general case (48) falls off as $1 / R^{3}$, so that a homogeneous Sommerfeld condition introduces an error which is vanishingly small for increasing $R$. As an example, for the dipole solution

$$
\Phi_{\text {Dipole }}=\partial_{z} \frac{f(t-r)}{r}=-\left(\frac{f^{\prime}(t-r)}{r}+\frac{f(t-r)}{r^{2}}\right) \cos \theta
$$


we have

$$
q=\frac{f(t-r) \cos \theta}{R^{3}}
$$

A homogeneous Sommerfeld condition at $r=R$ would lead to a solution $\tilde{\Phi}_{\text {Dipole }}$ containing a reflected ingoing wave. For large $R$,

$$
\tilde{\Phi}_{\text {Dipole }} \sim \Phi_{\text {Dipole }}+\kappa \frac{F(t+r-2 R) \cos \theta}{r},
$$

where $\partial_{t} f(t)=F(t)$ and the reflection coefficient has asymptotic behavior $\kappa=O\left(1 / R^{2}\right)$. More precisely, the Fourier mode

$$
\tilde{\Phi}_{\text {Dipole }}(\omega)=\partial_{z}\left(\frac{e^{i \omega(t-r)}}{r}+\kappa_{\omega} \frac{e^{i \omega(t+r-2 R)}}{r}\right),
$$

satisfies the homogeneous boundary condition $\left.\left(\partial_{t}+\partial_{r}\right)\left(r \tilde{\Phi}_{\text {Dipole }}\right)(\omega)\right|_{R}=0$ with reflection coefficient

$$
\kappa_{\omega}=\frac{1}{2 \omega^{2} R^{2}+2 i \omega R-1} \sim \frac{1}{2 \omega^{2} R^{2}} .
$$

Note that (50) and (49) satisfy

$$
\kappa \sim q R .
$$

In the case of a system of equations $\kappa$ will have $N$ components corresponding to the number of modes generated in the reflected wave. The boundary conditions lead to a system of simultaneous equations relating $\kappa$ to the components of the Sommerfeld data $q$. If these equations are nondegenerate then (51) continues to hold. However, degeneracies could conceivably lead to weaker asymptotic falloff of $\kappa$. (It would be interesting to determine whether such cases exist.) In any case, (51) gives the optimum allowable behavior of the reflection coefficients so that the asymptotic behavior of the Sommerfeld data $q$ is a good indicator of the quality of the boundary condition. This forms the basis of our investigation of the Maxwell and linearized Einstein equations with a spherical boundary in Sects. IV B and IV C.

A. A plane boundary. The key ideas in the above example are that (i) the Sommerfeld condition is only satisfied exactly by waves traveling in the radial direction and (ii) in the asymptotic limit $r \rightarrow \infty$ all waves from an isolated system propagate in the radial direction. This allows us to reformulate our discussion of the Sommerfeld condition by considering a wave $\Phi$ propagating in the domain $x<0$, which is incident on a plane boundary at $x=0$ with the boundary condition

$$
\left.K^{\alpha} \partial_{\alpha} \Phi\right|_{x=0}=0
$$

where $K^{\alpha} \partial_{\alpha}=\partial_{t}+\partial_{x}$ is the characteristic direction determined by the outward normal to the boundary $\partial_{x}$ and the time direction $\partial_{t}$. This homogeneous condition is satisfied for plane waves $\Phi=G\left(t+k_{x} x+k_{y} y+k_{z} z\right)$ incident on the boundary only for the single case $\left(k_{x}, k_{y}, k_{z}\right)=(1,0,0)$, i.e. a plane wave propagating in the outgoing normal direction. Plane waves in the normal direction pass through the boundary, whereas plane 
waves incident in other directions on the boundary give rise to a reflected wave. We will take advantage of this simplification of the plane wave case in discussing boundary conditions for electromagnetic and gravitational waves. The results then suggest how to formulate boundary conditions for an isolated electromagnetic or gravitational system with a spherical boundary of radius $R$, where in the limit $R \rightarrow \infty$ all radiation is incident normally.

For the electromagnetic case, we describe the field by means of a vector potential $A^{\mu}$ satisfying the Lorentz gauge condition. Maxwell's equations in a flat spacetime with Minkowski metric $\eta^{\mu \nu}$ then reduce to the wave equations

$$
\eta^{\alpha \beta} \partial_{\alpha} \partial_{\beta} A^{\mu}=0
$$

subject to the constraint

$$
C:=\partial_{\mu} A^{\mu}=0
$$

introduced by the Lorentz gauge condition. This constraint keeps us from requiring that each component of $A^{\mu}$ satisfy a homogeneous Sommerfeld condition, in contrast to the scalar example. The electromagnetic case also differs from the scalar case because of the remaining gauge freedom allowed by the Lorentz condition.

An electromagnetic plane wave incident in the outgoing normal direction can be described by the real part of the vector potential

$$
A_{\mu}=F(t-x) Q_{\mu}+G(t-x) K_{\mu},
$$

where $F(t-x)$ is complex, $Q^{\mu}=Y^{\mu}+i Z^{\mu}$ is a complex null polarization vector, $G(t-x)$ represents gauge freedom and $K^{\mu}=T^{\mu}+X^{\mu}$, in terms of the orthonormal tetrad $\left(T^{\mu}, X^{\mu}, Y^{\mu}, Z^{\mu}\right)$ aligned with the coordinate axes satisfying

$$
\eta_{\mu \nu}=-T_{\mu} T_{v}+X_{\mu} X_{v}+Y_{\mu} Y_{v}+Z_{\mu} Z_{v} .
$$

In order to formulate a gauge invariant boundary condition we consider the corresponding electromagnetic field tensor

$$
F_{\mu \nu}=\partial_{\mu} A_{\nu}-\partial_{\nu} A_{\mu}=-F^{\prime}(t-x)\left(K_{\mu} Q_{\nu}-Q_{\mu} K_{\nu}\right) .
$$

Here we adopt the notation $\partial_{u} F(u)=F^{\prime}(u)$. For this plane wave, all components of $F_{\mu \nu}$ satisfy

$$
K^{\mu} F_{\mu \nu}=0 .
$$

However, this condition rules out the possibility of a static electric field oriented normal to the boundary. For the purpose of formulating a boundary condition which only restricts propagating waves it suffices to consider the weaker condition

$$
K^{\mu} Q^{\nu} F_{\mu \nu}=0 .
$$

In terms of the electric and magnetic field components tangential to the boundary, (52) corresponds to the plane wave relations $\mathbf{E}_{\text {tan }} \cdot \mathbf{B}_{\text {tan }}=0$ and $\left|\mathbf{E}_{\text {tan }}\right|=\left|\mathbf{B}_{\text {tan }}\right|$, with the corresponding Poynting vector in the outward normal direction.

We can incorporate (52) into the following homogeneous Sommerfeld boundary conditions for the vector potential:

$$
\begin{gathered}
K^{v} K^{\mu} \partial_{\mu} A_{v}=0, \\
Q^{v} K^{\mu} \partial_{\mu} A_{v}=K^{v} Q^{\mu} \partial_{\mu} A_{\nu} .
\end{gathered}
$$


The remaining boundary condition can be expressed in Sommerfeld form by rewriting the constraint as

$$
C=\frac{1}{2}\left(-L^{v} K^{\mu}-K^{v} L^{\mu}+Q^{v} \bar{Q}^{\mu}+\bar{Q}^{v} Q^{\mu}\right) \partial_{\mu} A_{v}=0
$$

where $L^{\mu}=T^{\mu}-X^{\mu}$. Here $\left(K^{\mu}, L^{\mu}, Q^{\mu}\right)$ form a null tetrad according to the conventions

$$
\eta_{\mu \nu}=-K_{(\mu} L_{v)}+Q_{(\mu} \bar{Q}_{v)}
$$

We assume throughout the following that the spin transformation freedom $Q^{\mu} \rightarrow e^{i \alpha} Q^{\mu}$ has been restricted according to $K^{\mu} \partial_{\mu} \alpha=0$. The Sommerfeld boundary conditions (53), (54) and (55) have the required hierarchical, upper triangular form for a well posed IBVP, see Lemma 1.

For the purpose of extending this approach to the gravitational case, we write the linearized Einstein vacuum equations in the form

$$
\eta^{\alpha \beta} \partial_{\alpha} \partial_{\beta} \gamma^{\mu \nu}=0
$$

subject to the harmonic constraints

$$
C^{v}:=-\partial_{\mu} \gamma^{\mu \nu}=0
$$

Here, to linearized accuracy, we set $\sqrt{-g} g^{\mu \nu}=\eta^{\mu \nu}+\gamma^{\mu \nu}$ so that $\gamma_{\mu \nu}=-h_{\mu \nu}+\frac{1}{2} \eta_{\mu \nu} h$ represents the densitized version of the metric perturbation $g_{\mu \nu}=\eta_{\mu \nu}+h_{\mu \nu}$. (Indices of linearized objects are raised and lowered with the Minkowski metric.) The corresponding linearized curvature tensor is

$$
2 R_{\mu \nu \rho \sigma}=\partial_{\rho} \partial_{\nu} h_{\mu \sigma}-\partial_{\sigma} \partial_{\nu} h_{\mu \rho}-\partial_{\rho} \partial_{\mu} h_{\nu \sigma}+\partial_{\sigma} \partial_{\mu} h_{\nu \rho} .
$$

In the linear approximation, the diffeomorphism freedom reduces to the gauge freedom $h_{\mu \nu} \rightarrow h_{\mu \nu}+2 \partial_{(\mu} \xi_{v)}$, which leaves $R_{\mu \nu \rho \sigma}$ invariant.

A plane wave incident on the boundary in the outgoing normal direction is given by the real part of

$$
h_{\mu \nu}=F(t-x) Q_{\mu} Q_{v}+2 \partial_{(\mu} \xi_{v)}(t-x)=F(t-x) Q_{\mu} Q_{v}-2 K_{(\mu} \xi_{v)}(t-x),(60)
$$

where the $\xi_{v}(t-x)$ term describes a pure gauge wave. Similarly, a plane wave incident on the boundary in the ingoing normal direction is given by

$$
h_{\mu \nu}=F(t+x) Q_{\mu} Q_{\nu}-2 L_{(\mu} \xi_{v)}(t+x) \text {. }
$$

In these plane waves, $F$ describes the gravitational radiation. The curvature tensors corresponding to (60) and (61) are, respectively,

$$
R_{\mu \nu \rho \sigma}=2 F^{\prime \prime}(t-x) K_{[\mu} Q_{\nu]} Q_{[\rho} K_{\sigma]}
$$

and

$$
R_{\mu \nu \rho \sigma}=2 F^{\prime \prime}(t+x) L_{[\mu} Q_{\nu]} Q_{[\rho} L_{\sigma]} .
$$


The analogue of the boundary conditions (14),(15),(16) and (17) for a plane boundary are

$$
\begin{aligned}
K^{\mu} K^{\rho} K^{\sigma} \partial_{\mu} h_{\rho \sigma} & =-q_{K K}, \\
K^{\mu} K^{\rho} L^{\sigma} \partial_{\mu} h_{\rho \sigma} & =-q_{Q \bar{Q}}, \\
K^{\mu} K^{\rho} Q^{\sigma} \partial_{\mu} h_{\rho \sigma} & =-q_{K Q}, \\
\left(K^{\mu} Q^{\rho} Q^{\sigma}-Q^{\mu} Q^{\rho} K^{\sigma}\right) \partial_{\mu} h_{\rho \sigma} & =-q_{Q Q} .
\end{aligned}
$$

The outgoing plane wave (60) satisfies the homogeneous boundary conditions $q_{K K}=$ $q_{Q \bar{Q}}=q_{K Q}=q_{Q Q}=0$. For the ingoing plane wave (61),

$$
\begin{aligned}
& q_{K K}=-8 K^{\sigma} \xi_{\sigma}^{\prime}(t+x), \\
& q_{Q \bar{Q}}=-4 L^{\sigma} \xi_{\sigma}^{\prime}(t+x), \\
& q_{K Q}=-4 Q^{\sigma} \xi_{\sigma}^{\prime}(t+x), \\
& q_{Q Q}=-4 \bar{F}^{\prime}(t+x),
\end{aligned}
$$

all evaluated on the boundary at $x=0$. Thus the boundary conditions (64),(65) and (66) control the gauge waves entering through the boundary; and the condition (67) controls the gravitational waves entering.

In order to formulate a boundary condition with gauge invariant meaning analogous to (52) in the Maxwell case, we consider the linearized curvature tensor. Outgoing wave boundary conditions on the curvature tensor could be imposed by requiring that the Newman-Penrose component $\Psi_{0}=K^{\mu} Q^{v} Q^{\rho} K^{\sigma} R_{\mu \nu \rho \sigma}$ vanish on the boundary. (See [2] for a discussion of the appropriateness of this boundary condition.) However, this requirement involves second derivatives in the normal direction when expressed in terms of $\gamma_{\mu \nu}$. Instead, we require $\Psi:=K^{\mu} Q^{\nu} Q^{\rho} T^{\sigma} R_{\mu \nu \rho \sigma}=0$ on the boundary. The condition $\Psi=0$ is equivalent to $\Psi_{0}=0$ if the Ricci component $R_{\mu \nu} Q^{\mu} Q^{\nu}=0$, e.g. if the vacuum Einstein equations are satisfied.

A straightforward calculation leads to

$$
\begin{aligned}
-2 \Psi= & K^{\mu} Q^{\nu} Q^{\rho} T^{\sigma}\left(\partial_{\rho} \partial_{\nu} \gamma_{\mu \sigma}-\partial_{\sigma} \partial_{\nu} \gamma_{\mu \rho}-\partial_{\rho} \partial_{\mu} \gamma_{\nu \sigma}+\partial_{\sigma} \partial_{\mu} \gamma_{\nu \rho}\right)+\frac{1}{2} Q^{\nu} Q^{\rho} \partial_{\nu} \partial_{\rho} \gamma \\
= & K^{\mu} Q^{\nu} Q^{\rho} T^{\sigma}\left(-\partial_{\sigma} \partial_{\nu} \gamma_{\mu \rho}-\partial_{\rho} \partial_{\mu} \gamma_{\nu \sigma}+\partial_{\sigma} \partial_{\mu} \gamma_{\nu \rho}\right) \\
& +\frac{1}{2}\left(K^{\mu} K^{\sigma}+Q^{\mu} \bar{Q}^{\sigma}\right) Q^{\nu} Q^{\rho} \partial_{\nu} \partial_{\rho} \gamma_{\mu \sigma} \\
= & Q^{\nu} \partial_{\nu}\left(\frac{1}{2}\left(K^{\mu} K^{\sigma}+Q^{\mu} \bar{Q}^{\sigma}\right) Q^{\rho} \partial_{\rho} \gamma_{\mu \sigma}-K^{\mu} Q^{\rho} T^{\sigma} \partial_{\mu} \gamma_{\sigma \rho}\right) \\
& +T^{\sigma} \partial_{\sigma}\left(-K^{\mu} Q^{\nu} Q^{\rho} \partial_{\nu} \gamma_{\mu \rho}+K^{\mu} Q^{\nu} Q^{\rho} \partial_{\mu} \gamma_{\nu \rho}\right) .
\end{aligned}
$$

Thus, besides containing no second derivatives normal to the boundary, the condition $\Psi=0$ can be reduced to two first order conditions by factoring out the $Q^{\nu} \partial_{\nu}$ and $T^{\sigma} \partial_{\sigma}$ derivatives in (68) which are tangential to the boundary. There are many ways this can be done. In order to obtain first order conditions which fit into a hierarchy of Sommerfeld 
conditions, we modify (68) according to the steps

$$
\begin{aligned}
-2 \Psi= & Q^{\nu} \partial_{\nu}\left(\frac{1}{2}\left(K^{\mu} K^{\sigma}+Q^{\mu} \bar{Q}^{\sigma}\right) Q^{\rho} \partial_{\rho} \gamma_{\mu \sigma}-\frac{1}{2} K^{\rho} Q^{\mu} L^{\sigma} \partial_{\rho} \gamma_{\mu \sigma}\right. \\
& \left.-\frac{1}{2} K^{\mu} Q^{\rho} K^{\sigma} \partial_{\mu} \gamma_{\sigma \rho}\right) \\
& +T^{\sigma} \partial_{\sigma}\left(-K^{\mu} Q^{\nu} Q^{\rho} \partial_{\nu} \gamma_{\mu \rho}+K^{\mu} Q^{\nu} Q^{\rho} \partial_{\mu} \gamma_{\nu \rho}\right) \\
= & \frac{1}{2} Q^{\nu} \partial_{\nu}\left(\left(K^{\mu} K^{\sigma} Q^{\rho}+Q^{\mu} K^{\sigma} L^{\rho}-Q^{\mu} Q^{\sigma} \bar{Q}^{\rho}\right) \partial_{\rho} \gamma_{\mu \sigma}\right. \\
& \left.-2 Q^{\mu} C_{\mu}-K^{\mu} Q^{\rho} K^{\sigma} \partial_{\mu} \gamma_{\sigma \rho}\right) \\
& +T^{\sigma} \partial_{\sigma}\left(-K^{\mu} Q^{\nu} Q^{\rho} \partial_{\nu} \gamma_{\mu \rho}+K^{\mu} Q^{\nu} Q^{\rho} \partial_{\mu} \gamma_{\nu \rho}\right) \\
= & \frac{1}{2} Q^{\nu} \partial_{\nu}\left(\left(K^{\mu} K^{\sigma} Q^{\rho}-Q^{\mu} Q^{\sigma} \bar{Q}^{\rho}\right) \partial_{\rho} \gamma_{\mu \sigma}-2 K^{\mu} Q^{\rho} K^{\sigma} \partial_{\mu} \gamma_{\sigma \rho}-2 Q^{\mu} C_{\mu}\right) \\
& +T^{\sigma} \partial_{\sigma}\left(K^{\mu} Q^{\nu} Q^{\rho} \partial_{\mu} \gamma_{\nu \rho}\right) .
\end{aligned}
$$

Thus since the derivatives $Q^{v} \partial_{v}$ and $T^{v} \partial_{v}$ are tangential to the boundary, we can enforce $\Psi=0$ on the boundary through the first order boundary conditions

$$
\begin{gathered}
Q^{\alpha} Q^{\beta} K^{\mu} \partial_{\mu} \gamma_{\alpha \beta}=0, \\
K^{\alpha} Q^{\beta} K^{\mu} \partial_{\mu} \gamma_{\alpha \beta}-\frac{1}{2} K^{\alpha} K^{\beta} Q^{\mu} \partial_{\mu} \gamma_{\alpha \beta}+\frac{1}{2} Q^{\alpha} Q^{\beta} \bar{Q}^{\mu} \partial_{\mu} \gamma_{\alpha \beta}=0 .
\end{gathered}
$$

These two boundary conditions can then be included in a hierarchical set of Sommerfeld boundary conditions, according to the example

$$
\begin{aligned}
K^{\alpha} K^{\beta} K^{\mu} \partial_{\mu} \gamma_{\alpha \beta} & =0, \\
Q^{\alpha} Q^{\beta} K^{\mu} \partial_{\mu} \gamma_{\alpha \beta} & =0, \\
Q^{\alpha} \bar{Q}^{\beta} K^{\mu} \partial_{\mu} \gamma_{\alpha \beta} & =0, \\
K^{\alpha} Q^{\beta} K^{\mu} \partial_{\mu} \gamma_{\alpha \beta}-\frac{1}{2} K^{\alpha} K^{\beta} Q^{\mu} \partial_{\mu} \gamma_{\alpha \beta} & +\frac{1}{2} Q^{\alpha} Q^{\beta} \bar{Q}^{\mu} \partial_{\mu} \gamma_{\alpha \beta}=0 .
\end{aligned}
$$

The constraints $C_{\rho}=0$, which determine the remaining boundary conditions, can be cast in the Sommerfeld form

$$
C_{\rho}=\frac{1}{2}\left(L^{\nu} K^{\mu}+K^{v} L^{\mu}-\bar{Q}^{v} Q^{\mu}-Q^{\nu} \bar{Q}^{\mu}\right) \partial_{\mu} \gamma_{\nu \rho}=0
$$

which can also be incorporated into the hierarchy.

However, there are many alternative possibilities to (74)-(77) which preserve the hierarchical Sommerfeld structure and lead to a well posed IBVP. In the absence of a clear geometric approach, we next examine the boundary conditions appropriate to an isolated system by considering the resulting reflection off a spherical boundary.

B. Application to Maxwell fields with a spherical boundary. In the case of a general retarded solution for a massless scalar wave equation, we found that a Sommerfeld boundary condition on a spherical boundary of radius $R$ required data $q=O\left(1 / R^{3}\right)$. Homogeneous Sommerfeld data gave rise to an ingoing wave with reflection coefficient $\kappa=O\left(1 / R^{2}\right)$, as in (50). This is the best that can be achieved with a local first order 
homogeneous boundary condition on a spherical boundary. We now investigate the corresponding result for the constrained Maxwell equations expressed in terms of a vector potential $A^{\mu}$.

In doing so, we associate spherical coordinates $\left(r, x^{A}\right), x^{A}=(\theta, \phi)$, in a standard way with the Cartesian coordinates $x^{i}=(x, y, z)$, e.g. $z=r \cos \theta$. As in (56) we introduce a null tetrad $\left(K^{\mu}, L^{\mu}, Q^{\mu}\right)$ adapted to the boundary, where now $K^{\mu} \partial_{\mu}=\partial_{t}+\partial_{r}$, $L^{\mu} \partial_{\mu}=\partial_{t}-\partial_{r}$, and we fix the spin-rotation freedom in the complex null vector $Q^{\mu}=\left(0, Q^{i}\right)$ by setting

$$
Q^{i}=\frac{\partial x^{i}}{\partial x^{A}} Q^{A}
$$

where

$$
Q^{A}=\left(Q^{\theta}, Q^{\phi}\right)=\frac{1}{r}\left(1, \frac{i}{\sin \theta}\right) .
$$

We describe outgoing waves in terms of the retarded time $u=t-r$.

In order to investigate the vector potential describing the exterior radiation field emitted by an isolated system we introduce a Hertz potential with the symmetry

$$
H^{\mu \nu}=H^{[\mu \nu]}+\frac{1}{4} \eta^{\mu \nu} H
$$

Then the vector potential

$$
A^{\mu}=\partial_{\nu} H^{\mu \nu}
$$

satisfies the Lorentz gauge condition and generates a solution of Maxwell's equations provided the Hertz potential satisfies the wave equation. The trace $H$ represents pure gauge freedom.

We consider outgoing dipole waves oriented with the $z$-axis. Other dipole waves can be generated by a rotation. Higher multipole waves can be generated by taking spatial derivatives.

The choice $H=Z^{\alpha} \partial_{\alpha} \frac{F(u)}{r}, H^{[\mu \nu]}=0$ gives rise to the dipole gauge wave

$$
\begin{aligned}
A_{\mu}= & \left(\frac{F^{\prime \prime}(u)}{r}+\frac{F^{\prime}(u)}{r^{2}}\right) \cos \theta K_{\mu}+\left(\frac{2 F^{\prime}(u)}{r^{2}}+\frac{3 F(u)}{r^{3}}\right) \\
& \times \cos \theta \partial_{\mu} r-\left(\frac{F^{\prime}(u)}{r^{2}}+\frac{F(u)}{r^{3}}\right) Z_{\mu}
\end{aligned}
$$

with components

$$
\begin{aligned}
K^{\mu} A_{\mu} & =\left(\frac{F^{\prime}(u)}{r^{2}}+\frac{2 F(u)}{r^{3}}\right) \cos \theta \\
Q^{\mu} A_{\mu} & =\left(\frac{F^{\prime}(u)}{r^{2}}+\frac{F(u)}{r^{3}}\right) \sin \theta .
\end{aligned}
$$

In Appendix $\mathrm{V}$ we give some useful formulae underlying the calculation leading to (79) and the following results. 
The choice $H^{\mu \nu}=\left(T^{\mu} Z^{v}-Z^{\mu} T^{v}\right) \frac{f(u)}{r}$ gives rise to a dipole electromagnetic wave

$$
A_{\mu}=-\left(\frac{f^{\prime}(u)}{r}+\frac{f(u)}{r^{2}}\right) T_{\mu} \cos \theta-\frac{f^{\prime}(u)}{r} Z_{\mu}
$$

with components

$$
\begin{aligned}
A^{\mu} K_{\mu} & =\frac{f(u)}{r^{2}} \cos \theta, \\
A^{\mu} Q_{\mu} & =\frac{f^{\prime}(u)}{r} \sin \theta .
\end{aligned}
$$

The choice $H^{\mu \nu}=\left(X^{\mu} Y^{\nu}-Y^{\mu} X^{\nu}\right) \frac{f(u)}{r}$ gives rise to a dipole electromagnetic wave with the dual polarization

$$
A_{\mu}=-\left(\frac{f^{\prime}(u)}{r}+\frac{f(u)}{r^{2}}\right)\left(\frac{y X_{\mu}}{r}-\frac{x Y_{\mu}}{r}\right)
$$

with components

$$
\begin{gathered}
A^{\mu} K_{\mu}=0, \\
A^{\mu} Q_{\mu}=i\left(\frac{f^{\prime}(u)}{r}+\frac{f(u)}{r^{2}}\right) \sin \theta .
\end{gathered}
$$

We wish to formulate boundary conditions which generalize the Sommerfeld hierarchy (53) and (54) to a spherical boundary of radius $R$ in a way which minimizes reflection. By inspection of (79), (80) and (81), we consider the choice

$$
\begin{gathered}
\frac{1}{r^{2}} K^{\mu} \partial_{\mu}\left(r^{2} K^{\nu} A_{\nu}\right)=q_{K}, \\
\frac{1}{r} K^{\mu} \partial_{\mu}\left(r Q^{\nu} A_{\nu}\right)-Q^{\mu} \partial_{\mu}\left(K^{\nu} A_{\nu}\right)=q_{Q},
\end{gathered}
$$

chosen to minimize the asymptotic behavior of the Sommerfeld data. As before, the constraint determines the remaining boundary condition as part of the Sommerfeld hierarchy.

For the dipole gauge wave (79),

$$
q_{K}=-\frac{2 F(u) \cos \theta}{R^{4}}, \quad q_{Q}=0
$$

for the dipole electromagnetic wave (80),

$$
q_{K}=0, \quad q_{Q}=\frac{f(u)}{R^{3}} \sin \theta
$$

and for the dual dipole electromagnetic wave (81)

$$
q_{K}=0, \quad q_{Q}=\frac{-i f(u)}{R^{3}} \sin \theta .
$$

Overall this implies $q_{K}=O\left(1 / R^{4}\right)$ and $q_{Q}=O\left(1 / R^{3}\right)$. We have checked that homogeneous Sommerfeld data leads to reflection coefficients with overall behavior $\kappa=O\left(1 / R^{2}\right)$ in accordance with $(51)$. 
Note that the relations (A1) and (A8) allow us to express (82) and (83) in the form

$$
\begin{gathered}
\frac{1}{r^{2}} K^{v} K^{\mu} \partial_{\mu}\left(r^{2} A_{\nu}\right)=q_{K}, \\
Q^{v} K^{\mu} \partial_{\mu} A_{v}-K^{v} Q^{\mu} \partial_{\mu} A_{v}=q_{Q},
\end{gathered}
$$

which correspond to (7) and (8) when $\partial_{\mu}$ is generalized to the connection $\nabla_{a}$ in a curved space background with $K^{a} \nabla_{a} r=1$. Here (85) is equivalent to the gauge invariant condition

$$
Q^{v} K^{\mu} F_{\mu \nu}=q_{Q}
$$

C. Application to linearized gravitational fields with a spherical boundary. The gravitational case is more complicated than the electromagnetic case because the geometry of the boundary is coupled with the boundary condition. Additionally, there are no gauge invariant quantities, analogous to (86) in the electromagnetic case, on which to base first order boundary conditions. We begin with a discussion of how to adapt to a curved boundary the first order version of the $\Psi$ boundary condition given in Sect. IV A for a plane boundary.

In the nonlinear treatment of a curved boundary with unit outer normal $N^{a}$ we can decompose the metric according to

$$
g_{a b}=\tau_{a b}+N_{a} N_{b},
$$

where $\tau_{a b}$ is the metric intrinsic to the time-like boundary. Let $D_{a}$ denote the covariant derivative associated with $\tau_{a b}$. The extrinsic curvature of the boundary is

$$
N_{a b}=\tau_{a}^{c} \nabla_{c} N_{b}
$$

We complete an orthonormal basis by setting

$$
\tau_{a b}=-T_{a} T_{b}+Q_{(a} \bar{Q}_{b)}
$$

in terms of a time-like vector $T^{a}$ and complex null vector $Q^{a}$ tangent to the boundary.

We decompose $\Psi:=K^{a} Q^{b} Q^{c} T^{d} R_{a b c d}=\Psi_{T}+\Psi_{N}$ and the Weyl component $\Psi_{0}=K^{a} Q^{b} Q^{c} K^{d} R_{a b c d}=\Psi_{T}+\Psi_{N}+2 \Psi_{T N}$, where $K^{a}=T^{a}+N^{a}$ and

$$
\begin{aligned}
\Psi_{T} & =T^{a} Q^{b} Q^{c} T^{d} R_{a b c d}, \\
\Psi_{N} & =N^{a} Q^{b} Q^{c} T^{d} R_{a b c d}, \\
\Psi_{T N} & =T^{a} Q^{b} Q^{c} N^{d} R_{a b c d} .
\end{aligned}
$$

When the vacuum Einstein equations are satisfied the Riemann curvature tensor may be replaced by the Weyl tensor whose symmetry implies $\Psi_{T N}=0$. Therefore, in this case, $\Psi=0$ implies the vanishing of the Newman-Penrose Weyl component $\Psi_{0}=0$.

A short calculation gives the embedding formulae

$$
\Psi_{N}=Q^{b} Q^{c} T^{d}\left(D_{d} N_{b c}-D_{b} N_{c d}\right)
$$

and

$$
\Psi_{T}=T^{a} Q^{b} Q^{c} T^{d}\left({ }^{(3)} R_{a b c d}-N_{a c} N_{b d}+N_{b c} N_{a d}\right),
$$


where ${ }^{(3)} R_{a b c d}$ is the intrinsic curvature to the boundary, i.e.

$$
T^{a} Q^{b} Q^{c} T^{d(3)} R_{a b c d}=Q^{b} Q^{c} T^{d}\left(D_{d} D_{c}-D_{c} D_{d}\right) T_{b} .
$$

(These are the embedding equations for the Cauchy problem corrected for the space-like character of the normal to the boundary.)

We now apply these results to a spherical boundary $r=R$ in linearized theory off a Minkowski background, i.e. $g_{\mu \nu}=\eta_{\mu \nu}+\epsilon h_{\mu \nu}$ in standard inertial coordinates $x^{\mu}$, where $\epsilon$ is the linearization parameter. We choose $T_{\mu}=\partial_{\mu} t+O(\epsilon)$ and $N_{\mu}=\partial_{\mu} r+O(\epsilon)$. Then $D_{\mu} T_{\nu}=O(\epsilon)$ and $N_{\mu \nu}=R^{-1} Q_{\mu \nu}+O(\epsilon)$, where $Q_{\mu \nu}=Q_{(\mu} \bar{Q}_{\nu)}$ is the metric of a 2 -sphere of radius $R$. We choose the basis to satisfy $T^{\mu} D_{\mu} T_{\nu}=0$ and $T^{\mu} D_{\mu} Q_{\nu}=0$, so that

$$
\Psi_{T}=T^{\mu} Q^{\nu} Q^{\rho} T^{\sigma(3)} R_{\mu \nu \rho \sigma}+O\left(\epsilon^{2}\right)=T^{\sigma} D_{\sigma}\left(Q^{\nu} Q^{\rho} D_{\rho} T_{v}\right)+O\left(\epsilon^{2}\right)
$$

and

$$
\begin{aligned}
\Psi_{N}= & T^{\sigma} D_{\sigma}\left(Q^{\nu} Q^{\rho} N_{\rho \nu}\right)-Q^{\rho} D_{\rho}\left(Q^{\nu} T^{\sigma} N_{\sigma \nu}\right)+\frac{1}{2} Q^{\rho}\left(D_{\rho} Q_{\mu}\right) \bar{Q}^{\mu} Q^{\nu} T^{\sigma} N_{\sigma \nu} \\
& +\frac{1}{R} Q^{\nu} Q^{\rho} D_{\rho} T_{\nu}+O\left(\epsilon^{2}\right) .
\end{aligned}
$$

Thus the boundary conditions

$$
\begin{aligned}
Q^{v} Q^{\rho}\left(N_{\rho v}+D_{\rho} T_{v}\right) & =0, \\
Q^{v} T^{\rho} N_{\rho v} & =0,
\end{aligned}
$$

imply to linearized accuracy that

$$
\Psi=\frac{1}{R} Q^{v} Q^{\rho} D_{\rho} T_{\nu}
$$

This gives a geometric formulation of the first differential order version of the requirement that $\Psi \rightarrow 0$ in the asymptotic limit $R \rightarrow \infty$. However, $\Psi_{0}=O\left(1 / R^{5}\right)$ in an asymptotically flat space-time, whereas (91) leads to $\Psi=O\left(1 / R^{2}\right)$. This is an indication that the boundary conditions (90) might lead to more reflection than desirable. Can this be remedied by the introduction of, say, lower order terms in the boundary conditions? We investigate this question in the context of a well posed IBVP based upon the harmonic version of the linearized Einstein equations (57) and (58), where $\gamma^{\mu \nu}=-h^{\mu \nu}+\frac{1}{2} \eta^{\mu \nu} h$.

For this purpose, we now consider linearized outgoing waves in the harmonic gauge which are incident on a spherical boundary. We model our discussion on the Maxwell case by using the gravitational analogue of a Hertz potential $H^{\mu \alpha \nu \beta}[33,34]$, which has the symmetries

$$
H^{\mu \alpha \nu \beta}=H^{[\mu \alpha] \nu \beta}=H^{\mu \alpha[\nu \beta]}=H^{\nu \beta \mu \alpha}
$$

and satisfies the flat space wave equation

$$
\partial^{\sigma} \partial_{\sigma} H^{\mu \alpha \nu \beta}=0 .
$$

Then the densitized metric perturbation

$$
\gamma^{\mu \nu}=\partial_{\alpha} \partial_{\beta} H^{\mu \alpha \nu \beta}
$$


satisfies the linearized Einstein equations in the harmonic gauge. Outgoing waves can be generated from the potential

$$
H^{\mu \alpha \nu \beta}=\frac{f^{\mu \alpha \nu \beta}(u)}{r},
$$

and its spatial derivatives.

The incidence of such an outgoing wave on a boundary $r=R$ leads to reflection, with the asymptotic falloff of the reflection coefficients depending upon the choice of boundary conditions. We limit our calculation of reflection coefficients to the case of outgoing quadrupole waves, which can be obtained from the Hertz potential

$$
H^{\mu \alpha \nu \beta}=K^{\mu \alpha \nu \beta} \frac{f(u)}{r},
$$

where $K^{\mu \alpha \nu \beta}$ is a constant tensor. (All higher multipoles can be constructed by taking spatial derivatives.) $K^{\mu \alpha \nu \beta}$ has 21 independent components. However, the choice $K^{\mu \alpha \nu \beta}=\epsilon^{\mu \alpha \nu \beta}$ leads to $\gamma^{\mu \nu}=0$ so there are only 20 independent waves. These can be further reduced to pure gauge waves, corresponding to the trace terms in $K^{\mu \alpha \nu \beta}$, e.g. $K^{\mu \alpha \nu \beta}=\eta^{\alpha \nu} \eta^{\beta \mu}-\eta^{\mu \nu} \eta^{\alpha \beta}$ leads to a monopole gauge wave. Linearized gravitational waves arise from the trace-free part of $K^{\mu \alpha \nu \beta}$. There are ten independent quadrupole gravitational waves, corresponding to spherical harmonics with $(\ell=2,-2 \leq m \leq 2)$ in the two independent polarization states. The other ten independent potentials comprise two monopole gauge waves, three dipole gauge waves and five quadrupole gauge waves, for which the linearized Riemann tensor vanishes. It suffices to consider the following examples of waves with quadrupole dependence aligned with the $z$-axis. Other quadrupole waves can be obtained by rotation and have similar asymptotic behavior. Reflection coefficients from the other monopole and dipole gauge waves are smaller and provide no further useful information. The Hertz potential (92) gives rise to the perturbation

$$
\gamma^{\mu \nu}=K^{\mu \alpha \nu \beta} \partial_{\alpha} \partial_{\beta} \frac{f(u)}{r} .
$$

Appendix V lists useful formula for the calculations underlying the following results.

1. Quadrupole-monopole gauge wave. The Hertz potential

$$
H^{\mu \alpha \nu \beta}=\left(Z^{\mu} \eta^{\alpha \nu} Z^{\beta}+Z^{v} \eta^{\beta \mu} Z^{\alpha}-Z^{\mu} \eta^{\alpha \beta} Z^{v}-Z^{\beta} \eta^{\nu \mu} Z^{\alpha}\right) \frac{f(u)}{r}
$$

gives rise to a combination monopole-quadrupole gauge wave with components

$$
\begin{aligned}
Q^{\alpha} Q^{\beta} \gamma_{\alpha \beta} & =-2\left(\frac{f^{\prime}(u)}{r^{2}}+\frac{f(u)}{r^{3}}\right) \sin ^{2} \theta, \\
Q^{\alpha} \bar{Q}^{\beta} \gamma_{\alpha \beta} & =-2\left(\frac{f^{\prime \prime}(u)}{r}+\frac{2 f^{\prime}(u)}{r^{2}}+\frac{2 f(u)}{r^{3}}\right) \cos ^{2} \theta, \\
K^{\alpha} Q^{\beta} \gamma_{\alpha \beta} & =-\frac{f(u)}{r^{3}} \sin \theta \cos \theta, \\
K^{\alpha} K^{\beta} \gamma_{\alpha \beta} & =2\left(\frac{f^{\prime}(u)}{r^{2}}+\frac{2 f(u)}{r^{3}}\right) \cos ^{2} \theta, \\
\gamma & =-\frac{2 f^{\prime \prime}(u)}{r} \cos ^{2} \theta+2\left(\frac{f^{\prime}(u)}{r^{2}}+\frac{f(u)}{r^{3}}\right)\left(1-3 \cos ^{2} \theta\right) .
\end{aligned}
$$


Here the $\sin ^{2} \theta$ dependence of the spin-weight 2 component $Q^{\alpha} Q^{\beta} \gamma_{\alpha \beta}$ is a pure ${ }_{2} Y_{20}$ spin-weighted spherical harmonic; the $\sin \theta \cos \theta$ dependence of the spin-weight $1 \mathrm{com}$ ponent $K^{\alpha} Q^{\beta} \gamma_{\alpha \beta}$ is a pure ${ }_{1} Y_{20}$ harmonic; and the remaining spin-weight 0 components are mixtures of $Y_{00}$ and $Y_{20}$.

2. Quadrupole gravitational wave. The trace-free Hertz potential

$$
\begin{aligned}
H^{\mu \alpha \nu \beta}= & \left(\left(T^{\mu} Z^{\alpha}-Z^{\mu} T^{\alpha}\right)\left(X^{v} Y^{\beta}-Y^{v} X^{\beta}\right)\right. \\
& \left.+\left(X^{\mu} Y^{\alpha}-Y^{\mu} X^{\alpha}\right)\left(T^{v} Z^{\beta}-Z^{\nu} T^{\beta}\right)\right) \frac{f(u)}{r}
\end{aligned}
$$

gives rise to a perturbation with $\gamma=0$ and components

$$
\begin{aligned}
& Q^{\alpha} Q^{\beta} \gamma_{\alpha \beta}=2 i \sin ^{2} \theta\left(\frac{f^{\prime \prime}(u)}{r}+\frac{f^{\prime}(u)}{r^{2}}\right), \\
& Q^{\alpha} \bar{Q}^{\beta} \gamma_{\alpha \beta}=0, \\
& K^{\alpha} Q^{\beta} \gamma_{\alpha \beta}=i \cos \theta \sin \theta\left(\frac{2 f^{\prime}(u)}{r^{2}}+\frac{3 f(u)}{r^{3}}\right), \\
& K^{\alpha} K^{\beta} \gamma_{\alpha \beta}=0,
\end{aligned}
$$

which have spin-weighted $\ell=2, m=0$ dependence.

3. Dual quadrupole gravitational wave The trace-free Hertz potential

$$
\begin{aligned}
H^{\mu \alpha \nu \beta}= & \left(\left(T^{\mu} Z^{\alpha}-Z^{\mu} T^{\alpha}\right)\left(T^{\nu} Z^{\beta}-Z^{\nu} T^{\beta}\right)-\left(X^{\mu} Y^{\alpha}-Y^{\mu} X^{\alpha}\right)\left(X^{v} Y^{\beta}-Y^{v} X^{\beta}\right)\right. \\
& \left.+\frac{1}{3}\left(\eta^{\mu \nu} \eta^{\alpha \beta}-\eta^{\mu \beta} \eta^{\nu \alpha}\right)\right) \frac{f(u)}{r},
\end{aligned}
$$

obtained from the dual of (94), gives gives rise to a perturbation with $\gamma=0$ and components

$$
\begin{aligned}
& Q^{\alpha} Q^{\beta} \gamma_{\alpha \beta}=2 \sin ^{2} \theta\left(\frac{f^{\prime \prime}(u)}{r}+\frac{f^{\prime}(u)}{r^{2}}+\frac{f(u)}{r^{3}}\right), \\
& Q^{\alpha} \bar{Q}^{\beta} \gamma_{\alpha \beta}=4\left(\cos ^{2} \theta-\frac{1}{3}\right)\left(\frac{f^{\prime}(u)}{r^{2}}+\frac{f(u)}{r^{3}}\right), \\
& K^{\alpha} Q^{\beta} \gamma_{\alpha \beta}=\cos \theta \sin \theta\left(\frac{2 f^{\prime}(u)}{r^{2}}+\frac{f(u)}{r^{3}}\right), \\
& K^{\alpha} K^{\beta} \gamma_{\alpha \beta}=2\left(\cos ^{2} \theta-\frac{1}{3}\right) \frac{f(u)}{r^{3}},
\end{aligned}
$$

which have spin-weighted $\ell=2, m=0$ dependence. 
4. Sommerfeld-type boundary conditions. Sommerfeld boundary conditions consistent with a well posed harmonic IBVP have wide freedom regarding (i) partial derivative terms consistent with the hierarchical upper triangular structure of the boundary condition and (ii) lower differential order terms. Here we consider three choices of boundary conditions and compare their reflection coefficients. One basic idea common to these choices has already been used in the scalar and Maxwell cases, i.e by inspecting the asymptotic behavior of the waves (93), (95) and (96) we use the property $K^{\alpha} \partial_{\alpha} f(u)=0$ to introduce the appropriate powers of $r$ that lead to the smallest asymptotic behavior in the resulting Sommerfeld data.

Our first choice of boundary conditions is the mathematically simplest choice

$$
\begin{aligned}
\frac{1}{r^{2}} K^{\alpha} K^{\beta} K^{\mu} \partial_{\mu}\left(r^{2} \gamma_{\alpha \beta}\right) & =q_{K K}, \\
\frac{1}{r} Q^{\alpha} Q^{\beta} K^{\mu} \partial_{\mu}\left(r \gamma_{\alpha \beta}\right) & =q_{Q Q}, \\
\frac{1}{r} Q^{\alpha} \bar{Q}^{\beta} K^{\mu} \partial_{\mu}\left(r \gamma_{\alpha \beta}\right) & =q_{Q \bar{Q}}, \\
\frac{1}{r^{2}} K^{\alpha} Q^{\beta} K^{\mu} \partial_{\mu}\left(r^{2} \gamma_{\alpha \beta}\right) & =q_{K Q} .
\end{aligned}
$$

This was the choice adopted in numerical tests verifying the stability of the harmonic IBVP with a plane boundary [14]. The powers of $r$ in (97)-(100) are based upon the leading asymptotic behavior of the components for the gauge wave (93) and the gravitational waves (95) and (96). These choices lead to boundary data with the asymptotic behavior

$$
\begin{aligned}
& q_{K K} \sim \frac{f(u)}{R^{4}}, \\
& q_{Q Q} \sim \frac{f^{\prime}(u)}{R^{3}}, \\
& q_{Q \bar{Q}} \sim \frac{f^{\prime}(u)}{R^{3}}, \\
& q_{K Q} \sim \frac{f(u)}{R^{4}} .
\end{aligned}
$$

Thus the behavior of $q_{Q Q}$ and $q_{Q \bar{Q}}$ imply that the resulting reflection coefficients have overall asymptotic dependence no weaker than $\kappa=O\left(1 / R^{2}\right)$.

Our second choice, which is partially suggested by the electromagnetic case (83) and leads to weaker reflection, consists of the modifications

$$
\begin{aligned}
\frac{1}{r^{2}} K^{\alpha} K^{\beta} K^{\mu} \partial_{\mu}\left(r^{2} \gamma_{\alpha \beta}\right) & =q_{K K}, \\
\frac{1}{r^{2}} K^{\alpha} Q^{\beta} K^{\mu} \partial_{\mu}\left(r^{2} \gamma_{\alpha \beta}\right) & =q_{K Q}, \\
\frac{1}{r^{2}} Q^{\alpha} \bar{Q}^{\beta} K^{\mu} \partial_{\mu}\left(r^{2} \gamma_{\alpha \beta}\right)-\frac{\gamma}{r} & =q_{Q \bar{Q}}, \\
Q^{\alpha} Q^{\beta} K^{\mu} \partial_{\mu} \gamma_{\alpha \beta}-Q^{\alpha} K^{\beta} Q^{\mu} \partial_{\mu} \gamma_{\alpha \beta} & =q_{Q Q} .
\end{aligned}
$$

Now $q_{\text {.. }} \sim f(u) / R^{4}$ for both gravitational quadrupole waves. For the gauge waves, $q_{Q \bar{Q}} \sim f^{\prime}(u) / R^{3}$. Using the Regge-Wheeler-Zerilli perturbative formulation and the 
metric reconstruction method described in [35] we have independently checked that this leads to reflection coefficients $\kappa=O\left(1 / R^{3}\right)$ for the gravitational waves and $\kappa=O\left(1 / R^{2}\right)$ for the gauge waves in accord with (51). After replacing $\gamma_{\mu \nu}=-h_{\mu \nu}+$ $\frac{h}{2} \eta_{\mu \nu}$, observing that $K^{\mu} \partial_{\mu} r=1$ and identifying $\partial_{\mu}$ with the connection $\stackrel{\circ}{\nabla}_{a}$ of the background metric $\stackrel{\circ}{g}_{a b}$, (101)-(104) correspond to the boundary conditions (14)-(17) discussed in Sect. I C.

Our third choice of boundary conditions, motivated by the first order version of the $\Psi_{0}$ boundary condition (77), is

$$
\begin{aligned}
K^{\mu} \partial_{\mu}\left(r^{2} K^{\alpha} K^{\beta} \gamma_{\alpha \beta}\right) & =q_{K K}, \\
K^{\mu} \partial_{\mu}\left(r Q^{\alpha} Q^{\beta} \gamma_{\alpha \beta}\right) & =q_{Q Q}, \\
K^{\mu} \partial_{\mu}\left(r Q^{\alpha} \bar{Q}^{\beta} \gamma_{\alpha \beta}\right) & =q_{Q \bar{Q}}, \\
\frac{1}{r^{2}} K^{\mu} \partial_{\mu}\left(r^{2} K^{\alpha} Q^{\beta} \gamma_{\alpha \beta}\right) & -\frac{1}{2} Q^{\mu} \partial_{\mu} K^{\alpha} K^{\beta} \gamma_{\alpha \beta}+\frac{1}{2} \bar{Q}^{\mu} Q^{\alpha} Q^{\beta} \partial_{\mu} \gamma_{\alpha \beta}=q_{K Q} .
\end{aligned}
$$

However, for the gravitational quadrupole wave (95), this leads to $q_{K} Q \sim f^{\prime \prime}(u) / R^{2}$ and so it results in much stronger reflection than the first two choices. Thus, as might have been anticipated by the discussion following (91), the first order version of the $\Psi$ boundary condition is not as effective as (104)-(101) in the case of a spherical boundary.

\section{Conclusion}

We have considered the IBVP for a coupled system of quasilinear wave equations and established (local in time) well posedness for a large class of boundary conditions. In particular, this allows for the formulation of a well posed IBVP for quasilinear wave systems in the presence of constraints on finite domains with artificial, nonreflecting boundaries. Therefore, we anticipate that our results will have application to a wide range of problems in computational physics. Furthermore, since our proof is based on a reduction to a symmetric hyperbolic system with maximal dissipative boundary conditions, it also lays the path for constructing stable finite difference discretizations for such systems.

Our work has been motivated by the importance of the computation of gravitational waves from the inspiral and merger of binary black holes, which has enjoyed some recent success [36-40]. At present, however, none of the simulations of the binary black hole problem have been based upon a well posed IBVP. The closest example is the harmonic approach of the Caltech-Cornell group [41-43] which incorporates the freezing $\Psi_{0}$ boundary condition in second order form and has been shown to be well posed in the generalized sense in the high frequency limit [16].

Our results have potential application to improving the binary black hole simulations. However, many of these simulations are carried out using the BSSN formulation [44,45] of Einstein's equations, which differs appreciably from the harmonic formulation considered here. Although our results constitute a complete analytic treatment of the IBVP for the harmonic formulation of Einstein's equations, the extension to the BSSN formulation is not immediately evident. For this purpose, it would be useful to reformulate the boundary data for the harmonic problem in terms of the intrinsic geometry and extrinsic curvature of the boundary, as has been done for the initial data for the Cauchy problem. Such a geometric reformulation remains an outstanding problem. 
Acknowelegements. The work of O. R. was supported in part by CONICET, SECYT-UNC and NSF Grant INT0204937 to Louisiana State University. The work of O. S. was supported in part by grant CIC 4.19 to Universidad Michoacana, PROMEP UMICH-PTC-195 from SEP Mexico and CONACyT grant No. 61173. The work of J. W. was supported by NSF grant PH-0553597 to the University of Pittsburgh. During the course of this research we have profited from many discussions with H. Friedrich.

\section{Appendix A: Some Useful Formulae}

Here we give a short summary of the formulae and conventions underlying the calculational results of Sects. IV B and IV C. We have

$$
\partial_{\alpha} f(u)=-f^{\prime}(u) K_{\alpha}, \quad u=t-r, \quad K^{\alpha} \partial_{\alpha} K_{\beta}=0,
$$

so that

$$
\begin{aligned}
\partial_{\alpha} \partial_{\beta} \frac{f(u)}{r}= & \frac{f^{\prime \prime}(u)}{r} K_{\alpha} K_{\beta}+\frac{f^{\prime}(u)}{r^{2}}\left(K_{\alpha} r_{\beta}+r_{\alpha} K_{\beta}\right)+\frac{2 f(u)}{r^{3}} r_{\alpha} r_{\beta} \\
& -\left(\frac{f^{\prime}(u)}{r}+\frac{f(u)}{r^{2}}\right) r_{\alpha \beta}
\end{aligned}
$$

and

$$
\begin{aligned}
K^{\mu} \partial_{\mu} \partial_{\alpha} \partial_{\beta} \frac{f(u)}{r}= & -\frac{f^{\prime \prime}(u)}{r^{2}} K_{\alpha} K_{\beta}-\frac{2 f^{\prime}(u)}{r^{3}}\left(K_{\alpha} r_{\beta}+r_{\alpha} K_{\beta}\right) \\
& -\frac{6 f(u)}{r^{4}} r_{\alpha} r_{\beta}+\left(\frac{2 f^{\prime}(u)}{r^{2}}+\frac{3 f(u)}{r^{3}}\right) r_{\alpha \beta},
\end{aligned}
$$

where $r_{\alpha}:=\partial_{\alpha} r$ and $r_{\alpha \beta}:=\partial_{\alpha} \partial_{\beta} r$. The spatial components are

$$
r_{i}=\frac{x_{i}}{r}=(\sin \theta \cos \phi, \sin \theta \sin \phi, \cos \theta), \quad r_{i j}=\frac{\delta_{i j}}{r}-\frac{x_{i} x_{j}}{r^{3}} .
$$

Our conventions for the polarization dyad give rise to the Cartesian components

$$
\left(Q^{x}, Q^{y}, Q^{z}\right)=(\cos \theta \cos \phi-i \sin \phi, \cos \theta \sin \phi+i \cos \phi,-\sin \theta),
$$

which satisfy

$$
\begin{aligned}
\left(Q^{x}\right)^{2}+\left(Q^{y}\right)^{2} & =-\sin ^{2} \theta, \quad Q^{x} \frac{y}{r}-Q^{y} \frac{x}{r}=-i \sin \theta \\
Q^{x} \frac{y}{r}+Q^{y} \frac{x}{r} & =\sin \theta\left(2 \cos \theta \cos \phi \sin \phi+i\left(\cos ^{2} \phi-\sin ^{2} \phi\right)\right)
\end{aligned}
$$

and

$$
Q^{j} r_{i j}=\frac{Q_{i}}{r}, \quad Q^{j} \partial_{j} Q^{i}=\frac{\cot \theta}{r} Q^{i}, \quad Q^{j} \partial_{j} \bar{Q}^{i}=-\frac{\cot \theta}{r} \bar{Q}^{i}-\frac{2 r^{j}}{r} .
$$

From these follow the necessary commutation relations such as

$$
\left[r Q^{\mu} \partial_{\mu}, K^{\nu} \partial_{\nu}\right]=0 .
$$




\section{References}

1. Sarbach, O.: Absorbing boundary conditions for Einstein's field equations. J. Phys. Conf. Ser. 91, 012005 (2007)

2. Friedrich, H., Nagy, G.: The initial boundary value problem for Einstein's vacuum field equations. Commun. Math. Phys. 201, 619-655 (1999)

3. Friedrichs, K.O.: Symmetric positive linear differential equations. Commun. Pure Appl. Math. 11, 333-418 (1958)

4. Lax, P.D., Phillips, R.S.: Local boundary conditions for dissipative symmetric linear differential operators. Commun. Pure Appl. Math. 13, 427-455 (1960)

5. Secchi, P.: Well-posedness of characteristic symmetric hyperbolic systems. Arch. Rat. Mech. Anal. 134, 155-197 (1996)

6. Kreiss, H.O., Winicour, J.: Problems which are well-posed in a generalized sense with applications to the Einstein equations. Class. Quant. Grav. 23, S405-S420 (2006)

7. Kreiss, H.O.: Initial boundary value problems for hyperbolic systems. Commun. Pure Appl. Math. 23, 277-298 (1970)

8. Taylor, M.E.: Partial Differential Equations II, Qualitative Studies of Linear Equations. BerlinHeidelberg-New York: Springer, 1996

9. Kreiss, H.O., Reula, O., Sarbach, O., Winicour, J.: Well-posed initial-boundary value problem for the harmonic Einstein equations using energy estimates. Class. Quant. Grav. 24, 5973-5984 (2007)

10. Rauch, J.B., Massey, F.J. III.: Differentiability of solutions to hyperbolic initial-boundary value problems. Trans. Am. Math. Soc. 189, 303-318 (1974)

11. Kreiss, H-O., Wu, L.: On the stability definition of difference approximations for the initial boundary value problem. Appl. Num. Math. 12, 213-227 (1993)

12. Kreiss, H-O., Scherer, G.: Method of lines for hyperbolic differential equations. SIAM J. Numer. Anal. 29, 640-646 (1992)

13. Babiuc, M.C., Szilagyi, B., Winicour, J.: Harmonic initial-boundary evolution in general relativity. Phys. Rev. D 73, 064017(1)-064017(23) (2006)

14. Babiuc, M.C., Kreiss, H.-O., Winicour, J.: Constraint-preserving Sommerfeld conditions for the harmonic Einstein equations. Phys. Rev. D 75, 044002(1)-044002(13) (2007)

15. Hawking, S.W., Ellis, G.F.R.: The Large Scale Structure of Space Time. Cambridge: Cambridge University Press, 1973

16. Ruiz, M., Rinne, O., Sarbach, O.: Outer boundary conditions for Einstein's field equations in harmonic coordinates. Class. Quant. Grav. 24, 6349-6378 (2007)

17. Geroch, R.: Partial differential equations of physics. In: General Relativity: Proceedings. Edited by G.S. Hall, J.R. Pulham. Edinburgh: IOP Publishing, 1996, p. 19

18. Kreiss, H.O., Lorenz, J.: Initial-Boundary Value Problems and the Navier-Stokes Equations. London-New York: Academic Press, 1989

19. Winicour, J.: Characteristic evolution and matching. Liv. Rev. Rela. 28, 10 (2005)

20. Engquist, B., Majda, A.: Absorbing boundary conditions for the numerical simulation of waves. Math. Comp. 31, 629-651 (1977)

21. Higdon, R.L.: Absorbing boundary conditions for difference approximations to the multi-dimensional wave equation. Math. Comput. 47(176), 437-459 (1986)

22. Trefethen, L.N., Halpern, L.: Well-posedness of one-way wave equations and absorbing boundary conditions. Math. Comput. 47, 421-435 (1986)

23. Blaschak, J., Kriegsmann, G.: A comparative study of absorbing boundary conditions. J. Comput. Phys. 77, 109-139 (1988)

24. Jiang, H., Wong, Y.S.: Absorbing boundary conditions for second order hyperbolic equations. J. Comput. Phys. 88(1), 205-231 (1990)

25. Renaut, R.A.: Absorbing boundary conditions, difference operators, and stability. J. Comput. Phys. 102(2), 236-251 (1992)

26. Hedstrom, G.W.: Nonreflecting boundary conditions for nonlinear hyperbolic systems. J. Comput. Phys. 30(2), E222-E237 (1979)

27. Givoli, D.: Non-reflecting boundary conditions. J. Comput. Phys. 94(1), 1-29 (1991)

28. Grote, M.J., Keller, J.B.: Nonreflecting boundary conditions for Maxwell's equations. J. Comput. Phys. 139(2), 327-342 (1998)

29. Bayliss, A., Turkel, E.: Radiation boundary conditions for wavelike equations. Commun. Pure Appl. Math. 33, 707-725 (1980)

30. Lau, S.R.: Analytic structure of radiation boundary kernels for blackhole perturbations. J. Math. Phys. 46, 102503(1)-102503(21) (2005)

31. Buchman, L.T., Sarbach, O.C.A.: Towards absorbing outer boundaries in general relativity. Class. Quant. Grav. 23, 6709-6744 (2006) 
32. Buchman, L.T., Sarbach, O.C.A.: Improved outer boundary conditions for Einstein's field equations. Class. Quant. Grav. 24, S307-S326 (2007)

33. Sachs, R., Bergmann, P.G.: Structure of particles in linearized gravitational theory. Phys. Rev. 112, 674-680 (1958)

34. Boardman, J., Bergmann, P.G.: Spherical gravitational waves. Phys. Rev. 115, 1318-1324 (1959)

35. Sarbach, O., Tiglio, M.: Gauge invariant perturbations of Schwarzschild black holes in horizon penetrating coordinates. Phys. Rev. D 64, 084016(1)-084016(15) (2001)

36. Pretorius, F.: Evolution of binary black-hole spacetimes. Phys. Rev. Lett. 95, 121101(1)-121101(4) (2005)

37. Campanelli, M., Lousto, C.O., Marronetti, P., Zlochower, Y.: Accurate evolutions of orbiting black-hole binaries without excision. Phys. Rev. Lett. 96, 111101(1)-111101(4) (2006)

38. Baker, J.G., Centrella, J., Choi, D.-I., Koppitz, M., van Meter, J.: Gravitational-wave extraction from an inspiraling configuration of merging black holes. Phys. Rev. Lett. 96, 111102(1)-111102(4) (2006)

39. Gonzalez, J.A., Sperhake, U., Bruegmann, B., Hannam, M., Husa, S.: Total recoil: the maximum kick from nonspinning black-hole binary inspiral. Phys. Rev. Lett. 98, 091101 (2007)

40. Szilagyi, B., Pollney, D., Rezzolla, L., Thornburg, J., Winicour, J.: An explicit harmonic code for blackhole evolution using excision. Class. Quant. Grav. 24, S275-S293 (2007)

41. Lindblom, L., Scheel, M.A., Kidder, L.E., Owen, R., Rinne, O.: A new generalized harmonic evolution system. Class. Quant. Grav. 23, S447-S462 (2006)

42. Rinne, O., Lindblom, L., Scheel, M.A.: Testing outer boundary treatments for the Einstein equations. Class. Quant. Grav. 24, 4053-4078 (2007)

43. Pfeiffer, H.P., Brown, D.A., Kidder, L.E., Lindblom, L., Lovelace, G., Scheel, M.A.: Reducing orbital eccentricity in binary black hole simulations. Class. Quant. Grav. 24, S59-S82 (2007)

44. Shibata, M., Nakamura, T.: Evolution of three-dimensional gravitational waves: Harmonic slicing case. Phys. Rev. D 52, 5428-5444 (1995)

45. Baumgarte, T.W., Shapiro, S.L.: On the numerical integration of Einstein's field equations. Phys. Rev. D 59, 024007(1)-024007(7) (1999)

Communicated by G. W. Gibbons 